\title{
Synthetic neuronal datasets for benchmarking directed functional connectivity metrics
}

João Rodrigues, Alexandre Andrade

Background: Datasets consisting of synthetic neural data generated with quantifiable and controlled parameters are a valuable asset in the process of testing and validating directed functional connectivity metrics. Considering the recent debate in the neuroimaging community concerning the use of these metrics for fMRI data, synthetic datasets that emulate the BOLD signal dynamics have played a central role by supporting claims that argue in favor or against certain choices. Generative models often used in studies that simulate neuronal activity, with the aim of gaining insight into specific brain regions and functions, have different requirements from the generative models for benchmarking datasets. Even though the latter must be realistic, there is a tradeoff between realism and computational demand that needs to be contemplated and simulations that efficiently mimic the real behavior of single neurons or neuronal populations are preferred, instead of more cumbersome and marginally precise ones. Methods: this work explores how simple generative models are able to produce neuronal datasets, for benchmarking purposes, that reflect the simulated effective connectivity and, how these can be used to obtain synthetic recordings of EEG and fMRI BOLD signals. The generative models covered here are AR processes, neural mass models consisting of linear and nonlinear stochastic differential equations and populations with thousands of spiking units. Forward models for EEG consist in the simple three-shell head model while the fMRI BOLD signal is modeled with the Balloon-Windkessel model or by convolution with a hemodynamic response function. Results: the simulated datasets are tested for causality with the original spectral formulation for Granger causality. Modeled effective connectivity can be detected in the generated data for varying connection strengths and interaction delays. Discussion: all generative models produce synthetic neuronal data with detectable causal effects although the relation between modeled and detected causality varies and less biophysically realistic models offer more control in causal relations such as modeled strength and frequency location. 
1 João Rodrigues ${ }^{\mathrm{a}}$, Alexandre Andrade ${ }^{\mathrm{a}}$

2 (a) Institute of Biophysics and Biomedical Engineering, Faculty of Sciences, University of 3 Lisbon, Campo Grande, 1749-016 Lisbon, Portugal.

4 Corresponding author: João Rodrigues

5 Email: jprodrigues@ fc.ul.pt Phone: +351931 105203 Address: Institute of

6 Biophysics and Biomedical Engineering, Faculty of Sciences, University of Lisbon, Campo

7 Grande, 1749-016 Lisbon, Portugal. 
1. Introduction

10 The field of brain research that studies connectivity relies in an ever evolving array of

11 methods that aim at finding directed or undirected connectivity links from recorded

12 neuronal datasets which pose different challenges such as having nonlinear relations, non-

13 stationary dynamics, low signal-to-noise ratio (SNR), linear mixes or slow dynamics.

14 Therefore, most studies that introduce or discuss connectivity metrics use not only real but

15 also simulated datasets for demonstration and validity purposes on a controlled

16 environment. These simulated datasets are obtained through generative models that

17 simulate the conception of neuronal activity, like local field potentials (LFPs), with a

18 certain degree of realism and can be followed by forward biophysical modeling where

19 LFPs are transformed into other neuronal recording datasets like electroencephalography

20 (EEG), magnetoencephalography or functional magnetic resonance imaging (fMRI) blood-

21 oxygen-level dependent (BOLD) signals, for example. With these controlled simulations it

22 is possible to isolate the effect of certain parameters and understand how directed

23 functional connectivity metrics perform in a realistic range of values.

24 As far as directed functional connectivity is concerned, Granger causality (GC) based

25 metrics have been in the center of several studies that discussed their plausibility in the

26 analysis of fMRI BOLD datasets due to the inherent low SNR, slow dynamics compared to

27 neuronal activity and confounding effects due to variable vascular latencies across brain

28 regions (Deshpande, Sathian \& Hu, 2009; Smith et al., 2010; Valdes-Sosa et al., 2011;

29 Seth, Chorley \& Barnett, 2013; Rodrigues \& Andrade, 2014). Most of these studies used

30 simulated datasets to support their claims with generative models such as networks of 'on-

31 off' neurons (Smith et al., 2010), multivariate vector autoregressive (MVAR) processes

32 with real LFPs (Deshpande, Sathian \& Hu, 2009; Rodrigues \& Andrade, 2014) and

33 columns of thousands of spiking units (Seth, Chorley \& Barnett, 2013) and BOLD forward

34 models consisting of linear operations or more biophysically realistic models. Other

35 problems such as the effect of volume conduction in directed connectivity has also been

36 addressed with LFPs being modeled as sinusoids and the forward EEG model as a linear

37 mix of different LFPs (Kaminski \& Blinowska, 2014). 
38 The objective of this work is to present and test the ability of popular generative and

39 forward models to produce synthetic neuronal datasets with modeled causal effects, to be

40 used as benchmarks.

41 2. Methods

42 In this section we succinctly present the methodological framework used in this study from

43 the modeling techniques to the connectivity metrics employed. Figure 1 depicts the

44 generative models to simulate LFPs and the forward models for FMRI BOLD signals and

45 EEG signals. The ensuing subsections expand on each technique separately by laying

46 theoretical foundations, mentioning available software and explaining why these

47 simulations have been important in the development of brain connectivity tools. Although

48 we present the generative model definitions in their general terms for any given network, in

49 this work simulations only encompass two nodes.

50 [INSERT FIGURE 1 HERE]

51 Simulations ran on a desktop PC equipped with an Intel ${ }^{\circledR}$ Core ${ }^{\mathrm{TM}}$ i7-2600K CPU

$52 @ 3.4 \mathrm{GHz}, 8 \mathrm{~GB}$ of RAM and an NVIDIA® GeForce ${ }^{\circledR}$ GTX 580 GPU with 3 GB graphics

53 memory. Simulations of Izhikevich columns ran on modified CUDA/C++ routines from

54 CARLsim 2.0 (Richert et al., 2011) and used the parallel processing capabilities of the

55 GPU. The remaining generative and forward models were implemented and ran in Matlab®

56 and used only the CPU without any explicit parallelization.

57 2.1. Autoregressive modeling

58 Autoregressive (AR) modeling is the simplest and most straightforward method for

59 simulating neuronal datasets. By specifying the MVAR equations and parameters it is

60 possible to simulate datasets from networks with different number of nodes, topology,

61 noise, interaction delays and duration. Although it has been shown that the AR model's

62 impulse response function has a transfer function that resembles the transfer function of a

63 simple physiological model of EEG generation (Blinowska \& Franaszczuk, 1989), as most

64 connectivity metrics are parametric, estimating an MVAR model for datasets generated by

65 linear AR processes might not pose the required challenge expected when presenting a

66 novel connectivity metric. Nevertheless, this method has been used in many well-known 
67 works either standalone or as initial validation, followed by real neurobiological or 68 neuroimaging datasets. It has been used to demonstrate that partial directed coherence 69 (PDC) can correctly identify the directed connectivity in multivariate datasets from 70 elaborate networks with reciprocal connections and interaction delays with different 71 magnitudes and durations (Baccalá \& Sameshima, 2001). Similarly, the direct directed 72 transfer function specific sensitivity to directed causal effects was also demonstrated with 73 data from a MVAR model with unitary delays between variables (Korzeniewska et al., 74 2003). Equivalent models have been used to further study metrics like directed transfer 75 function (DTF) (Eichler, 2006), improvements on spectral GC (Chen, Bressler \& Ding, 76 2006) or demonstration of connectivity metrics in a vastly used toolbox (Seth, 2010).

77 MVAR models have also been key in data simulation for the still open debate (Smith et al., 78 2010; Valdes-Sosa et al., 2011; Seth, Chorley \& Barnett, 2013) concerning the use of 79 directed connectivity metrics to assess causal influences from fMRI BOLD signals.

80 Roebroeck used synthetic data generated from a bi-dimensional first-order AR process as 81 LFPs which were later transformed into BOLD signals in which causality was assessed 82 with GC for different experimental parameters (Roebroeck, Formisano \& Goebel, 2005).

83 Later, Schippers used the same MVAR model to understand the effect of hemodynamic lag 84 opposing the interaction neuronal delay in group analysis (Schippers, Renken \& Keysers, 85 2011) and Barnett used a similar first order model to solve GC analytically after digital 86 filtering (Barnett \& Seth, 2011). Other studies used MVAR models to study the 87 effectiveness of GC applied to cluster sets obtained with independent component analysis 88 (Londei et al., 2006) and principal component analysis with partial canonical correlation 89 analysis (Sato et al., 2010). Other have used AR networks where real LFP propagate 90 according to predefined weights and delays to study the dependence of GC metrics to 91 experimental parameters (Deshpande, Sathian \& Hu, 2009; Rodrigues \& Andrade, 2014).

92 An MVAR model of order $p$ can be defined by $\mathbf{X}(t)=\sum_{j=1}^{p} \boldsymbol{\Phi}_{j} \mathbf{X}(t-j-\mathbf{D})+\mathbf{w}(t)$ where

$93 \mathbf{X}(t)$ is the multivariate time-series, $\boldsymbol{\Phi}_{j}$ is the MVAR coefficient matrix for time-lag $j, p$ is 94 the model order, $\mathbf{D}$ is the interaction delay matrix and $\mathbf{w}(t)$ is the innovation matrix 95 consisting in independent white noise. With these parameters it is possible to define all the 96 aforementioned network properties. 
97 Here, we also consider the possibility of defining the spectral peak frequency of the AR

98 process that generates the activity of each variable. Considering the AR model for variable

$99 n$, without accounting for inter-variable interactions, $X_{n}(t)=\sum_{j=1}^{p} \varphi_{n j} X_{n}(t-j)+w_{n}(t)$ a

100 spectral peak frequency can be identified if the model is causal which requires all the roots

101 of the AR polynomial $\varphi_{n j}(z)=1-\sum_{j=1}^{p} \varphi_{n j} z^{j}$ to be outside the unit circle. As proved by

102 (Jiru, 2008), when the absolute value of the AR coefficient for the time-lag $p$ is close to

103 unity, $\left|\varphi_{p}\right| \approx 1$, the spectral peak frequencies have approximately the same values as the

104 arguments of the roots of the AR polynomial. Other properties, specially for models with $p$

$105=2,3$ can be found in (Jiru, 2008). The possibility to manipulate the spectral peak

106 frequencies of each variable in the MVAR model adds up to the previous parameters so

107 now it is possible to generate synthetic data for a given number of variables with a known

108 connectivity pattern (reflected in the inter-variable AR parameters distribution), interaction

109 delays (reflected in $\mathbf{D}$ ), interaction strength (reflected in the inter-variable AR parameters

110 value), SNR (reflected in $\sigma_{w}^{2}$ ) and peak frequency (reflected in the intra-variable

111 parameters).

112 Although the inter-variable AR coefficients define the interaction strength between

113 variable, it is also possible to compute the required values for these parameters in order to

114 obtain a desired theoretical GC as done in (Barnett \& Seth, 2011). This is done for each

115 variable pair by analytically deriving the expression for their theoretical GC value, based in

116 the MVAR coefficient matrix, transfer function and noise covariance matrix, and solving it

117 for the inter-variable coefficients.

118 For our simulations, a bivariate AR(2) model was created with variable 1 exerting causal

119 effect in variable 2 at a specific frequency $\omega$, with variable intensity $F_{X_{1} \rightarrow X_{2}}$ and delay $d_{21}$

120 following the expression:

$$
\begin{aligned}
& X_{1}(t)=\varphi_{11}(2) X_{1}(t-2)+\varphi_{11}(1) X_{1}(t-1)+w_{1}(t) \\
& X_{2}(t)=\varphi_{22}(2) X_{2}(t-2)+\varphi_{22}(1) X_{2}(t-1)+\varphi_{21}(1) X_{1}\left(t-d_{21}\right)+w_{2}(t)
\end{aligned}
$$

121 Here $w_{1}$ and $w_{2}$ are the model's uncorrelated, zero mean, unit variance, white Gaussian

122 innovation processes. Causality was chosen to occur always in the gamma band, more 
123 specifically at $33 \mathrm{~Hz}$ so, the AR parameters for variable 1 AR process were chosen so the

124 spectral peak occurs at this frequency. Following the relationship $\omega= \pm \arccos \left(\varphi_{11}(1)\left(\varphi_{11}(2)-1\right) /\left(4 \varphi_{11}(2)\right)\right)$ between spectral peak frequency and AR

126

127

128

129

130

131

$$
\mathrm{F}_{X_{1} \rightarrow X_{2}}(\omega)=\ln \left(1+\frac{\varphi_{21}(2)^{2}}{\varphi_{11}(1)^{2}+\varphi_{11}(2)^{2}+2 \varphi_{11}(1) \cos (\omega)\left(\varphi_{11}(2)-1\right)-2 \varphi_{11}(2) \cos (2 \omega)+1}\right)
$$

132 Solving (2) for $\varphi_{21}(1)$ allows an AR(2) model to be built with the desired causality.

133 Although LFPs simulation might seem redundant (GC estimation from data modeled with

134 parameters obtained by the analytical solution of the spectral GC formulation) this data is

135 helpful to analyze the effects of the forward EEG and BOLD models and to benchmark or 136 interaction delays $\left(\mathrm{F}_{X_{1} \rightarrow X_{2}}(33 \mathrm{~Hz})=5, d_{21}=[4 \mathrm{~ms}\right.$, and 20 to $100 \mathrm{~ms}$ in steps of $\left.20 \mathrm{~ms}]\right)$.

139 Initial values were randomly assigned, the simulations produced $60 \mathrm{~s}$ of data and the first $14020 \mathrm{~s}$ were discarded to remove transient effects, data was generated at $1 \mathrm{k} \mathrm{Hz}$ and 141 subsampled to $250 \mathrm{~Hz}$. Each $60 \mathrm{~s}$ of data required $\sim 1 \mathrm{~s}$ of simulation time.

\section{2.2. Coupled oscillators}

143 Coupled oscillator networks are a more realistic way to represent the dynamics between 144 neuronal populations. Each node usually represents a population of excitatory and 145 146 inhibitory spiking neurons that exhibit oscillations with varying levels of synchrony in specific frequency ranges. The network can comply with one of two following dynamical

147 regimes: a synchronous state with self-sustained oscillations (Börgers \& Kopell, 2003) or in 148 an asynchronous state with transient oscillations (Mattia \& Del Giudice, 2002). These 
networks have been used to show that DTF can be interpreted within the GC framework (Kamiński et al., 2001) by modeling interacting cortical columns with excitatory and inhibitory populations with delay-coupled nonlinear stochastic differential equations (SDE). Similar SDE delayed networks, called Wilson-Cohen models, are also used in (Deco, Jirsa \& McIntosh, 2011) to study the dynamics of simulated resting state networks (RSNs). A similar but more detailed dynamic causal model is used to study GC (Friston et al., 2014) where each cortical column is comprised of pyramidal and inhibitory cells from supra-granular layers, excitatory spiny cells in granular layers and deep pyramidal cells in infra-granular layers.

A general network of coupled second order differential equations similar to (Kamiński et al., 2001), represents a node as a system with delay-coupled nonlinear SDE for the excitatory population defined by:

$$
\ddot{x}_{n}+(a+b) \dot{x}_{n}+a b x_{n}(t)=-k_{e i} S\left(y_{n}(t)\right)+\sum_{m=1}^{N} k_{n m} S\left(x_{m}\left(t-d_{n m}\right)\right)+I_{n}(t)+w_{x_{n}}(t)
$$

and inhibitory population defined by:

$$
\ddot{y}_{n}+(a+b) \dot{y}_{n}+a b y_{n}(t)=k_{i e} S\left(x_{n}(t)\right)+w_{y_{n}}(t)
$$

\section{Variables $x_{n}$ and $y_{n}$ represent the LFPs of excitatory and inhibitory populations} respectively of node $n, k_{e i}$ and $k_{i e}$ their respective coupling coefficients, $k_{n m}$ is the coupling coefficient from node $n$ to node $m, d_{n m}$ is the delay from node $m$ to node $n, a$ and $b$ are time constants that define the rate at which activity decays without input and, $w_{x_{n}}$ and $w_{y_{n}}$ are the independent white noise processes for nodes $n$ and $m$ respectively. $I_{n}(t)$ is the external input to the excitatory population and $S$ is the following sigmoidal function for a modulatory parameter $S_{m}$ :

$$
\begin{aligned}
& S\left(x, S_{m}\right)=\left\{\begin{array}{cc}
S_{m}\left(1-\mathrm{e}^{-\left(\mathrm{e}^{x}-1\right) / S_{m}}\right) & \text { if } x>-u_{0} \\
-1 & \text { if } x \leq-u_{0}
\end{array}\right. \\
& u_{0}=-\ln \left(1+\ln \left(1+1 / S_{m}\right)\right)
\end{aligned}
$$

Following the notations in (Freeman, 1987), (3) and (4) is a coupling between $K O_{i}$ and $K O_{e}$ subsets hence, each node can be seen a $K I_{e, i}$ set. In this work these parameters were used with the same values as in (Freeman, 1987) and (Kamiński et al., 2001): $a=0.22 / \mathrm{ms}$, 
$172 b=0.72 / \mathrm{ms}, k_{e i}=0.4, k_{i e}=0.1, S_{m}=5$ and the independent Gaussian white noise

173 processes had zero mean and 0.04 variance. The system's numerical solution was

174 approximated with a fourth order Runge-Kutta method (delays where linearly interpolated

175 for the intermediate increments) using a time-step of $0.1 \mathrm{~ms}$, with the noise term being

176 integrated with the Euler method using the same time-steps. The LFPs were initialized as

177 zero for as long as the longest delay present in the simulation required.

178 A network of two $K I_{e, i}$ sets, defined by the interactions in (3) and (4), was simulated for 60

$179 \mathrm{~s}$ with varying values of weak and strong coupling $\left(k_{21}=[0,0.1,0.2,0.5,0.7,1,3,5,10\right.$,

$\left.18015,22,30], d_{21}=20 \mathrm{~ms}\right)$ and interaction delay $\left(k_{21}=30, d_{21}=[4 \mathrm{~ms}\right.$ and 20 to $100 \mathrm{~ms}$ in

181 steps of $20 \mathrm{~ms}$ ] ) between sets 1 and 2. The first $20 \mathrm{~s}$ were discarded to remove transient

182 effects, data was generated at $10 \mathrm{k} \mathrm{Hz}$ and subsampled to $250 \mathrm{~Hz}$. External input $I_{n}(t)$

183 lasted 1 millisecond and had $1 \%$ probability of occurrence for each set. Each $60 \mathrm{~s}$ of data

184 required $\sim 181 \mathrm{~s}$ of simulation time.

185 As (Friston et al., 2014) concludes that GC is not appropriate for data generated by delay-

186 coupled oscillators with unstable modes and because self-sustained oscillations occur in

187 large scale simulations (Deco, Jirsa \& McIntosh, 2011), we also focus our simulations on

188 networks functioning in the synchronous mode. In this regime, since oscillators show a

189 limit cycle phase space trajectory this phase can be modeled by a single dynamical variable

190 reducing the former models to a simpler phase oscillators where it is possible to define the

191 oscillating frequency. Cabral et al. (Cabral et al., 2011) modeled RSNs with several

192 delayed-phase oscillator networks using the Kuramoto model (Kuramoto, 1984), layered

193 for each frequency of interest. Therefore, in this work, we use the same network building

194 scheme consisting of several stacked layers of two coupled oscillators (one layer for each

195 natural frequency) where each phase variable $\theta_{n}$ is governed by the following dynamical

196 equation:

$$
\dot{\theta}_{n}=\omega_{n}+k \sum_{m=1}^{N} C_{n m} \sin \left(\theta_{m}\left(t-d_{n m}\right)-\theta_{n}(t)\right)+w_{\theta_{n}}(t)
$$

197 Here, $\omega_{n}$ is the angular frequency of each oscillator $(\mathrm{rad} / \mathrm{s}), C_{n m}$ is the relative coupling

198 coefficient from node $m$ to node $n, k$ is a global coupling coefficient and the remaining 
variables represent the same parameters as in the previous dynamic equations. The neuronal activity can be obtained as the firing rate of the population of neurons represented by each oscillator. Following the procedure in (Cabral et al., 2011) the firing rate is the sine function $r_{n}=\sin \left(\theta_{n}(t)\right)$ of phase variable.

For our simulations the system's numerical solution was approximated with the EulerMaruyama (Kloeden \& Platen, 1992) method using a time-step of $0.1 \mathrm{~ms}$. Phases were initialized randomly in the initial instants corresponding to the longest delay. A network of two nodes was simulated for $60 \mathrm{~s}$ (first $20 \mathrm{~s}$ were discarded to remove transient effects) by having three independent layers of pairs of phase-delayed coupled Kuramoto oscillators where at each layer share the same natural frequency and follow the dynamics in (6). Layers 1,2 and 3 oscillate at 5, 33 and $60 \mathrm{~Hz}$ respectively and only layer 2 has a coupling coefficient between oscillators different than 0 . Therefore, phase-delayed coupling can only occur at the $33 \mathrm{~Hz}$ and from node 1 to 2 . Coupling varied from weak to strong ( $k=[0,0.1$, $\left.0.2,0.5,0.7,1,3,5,10,15,22,30], d_{21}=20 \mathrm{~ms}\right)$ and interaction delays followed the same values as the previous simulations $\left(k=30, d_{21}=[4 \mathrm{~ms}\right.$ and 20 to $100 \mathrm{~ms}$ in steps of 20 $\mathrm{ms}]$ ). Each $60 \mathrm{~s}$ of data required $\sim 55 \mathrm{~s}$ of simulation time.

\subsection{Izhikevich columns}

The former oscillator models can be simulated in greater detail by modeling their constituting spiking units individually. In the context of testing directed connectivity, this modeling was used in (Seth, Chorley \& Barnett, 2013) to understand how GC's sensitivity is affected by vascular latencies opposing the neuronal lag in BOLD signal time-series sampled with decreasing time repeat (TR). Besides spiking units, their synapses were also modeled with explicit NMDA, AMPA, GABAa and GABAb conductances and short-term plasticity (STP). The Izhikevich spiking model is able to produce several firing patterns observed in real neurons without the computational demand of more biophysically realistic models like the Hodgkin-Huxley which makes it appropriate for larger scale simulations (Izhikevich, 2003). This model replaces the bio-physiologic meaning of the HodgkinHuxley model's equations and parameters by a topologically equivalent phase dynamic modeled with two ordinary differential equations and four parameters: 


$$
\begin{aligned}
& \dot{v}=0.04 v^{2}+5 v+140-u+I \\
& \dot{u}=a(b v-u)
\end{aligned}
$$

228 And the additional after-spike resetting when $v \geq+30 \mathrm{mV}, v=c$ and $u=u+d$.

229 The variable $v$ represents the neuron's membrane potential and $u$ its recovery variable.

230 Parameters $a, b, c$ and $d$ are defined in order to implement one of the twenty spiking

231 neurons shown in (Izhikevich, 2004). Similarly to (Seth, Chorley \& Barnett, 2013), in this

232 work we simulate cortical columns with two different neurons: regular spiking excitatory

233 pyramidal neurons ( $a=0.02, b=0.2, c=-65, d=8$ ) and fast spiking inhibitory

234 interneurons ( $a=0.1, b=0.2, c=-65, d=2$ ). Due to the model's simplicity it is also

235 computationally amenable to compute the synaptic input to each neuron with the

236 neurotransmitter conductances for each receptor type $\left(g_{A M P A}, g_{N M D A}, g_{G A B A a}\right.$ and $\left.g_{G A B A b}\right)$ :

$$
I=g_{A M P A}(v-0)+g_{N M D A} \frac{[(v+80) / 60]^{2}}{1+[(v+80) / 60]^{2}}(v-0)+g_{G A B A a}(v-70)+g_{G A B A b}(v-90)
$$

237 These conductances are modeled with spike timing dependent plasticity (STDP) by being

238 affected by incoming spike's origin and timing. Therefore, spikes incoming from excitatory

239 neurons can change $g_{A M P A}$ and $g_{N M D A}$ while spikes incoming from inhibitory neurons can

240 change $g_{G A B A a}$ and $g_{G A B A b}$. This update depends upon the difference between the timing of

241 the post and pre-synaptic spikes, $\Delta t$, and two time constants for slow $(\tau=0.1 \mathrm{~ms})$ and fast

$242(\tau=0.01 \mathrm{~ms})$ synapses and is modeled exponentially: increment $\propto e^{-\Delta t / \tau}$ if $\Delta t>0$ and

243 decrement $\propto-e^{\Delta t / \tau}$ if $\Delta t \leq 0$.

244 STP is also modeled in the synaptic weights, influenced by pre-synaptic activity, with the 245 scale factor $s(t)$ :

$$
\begin{aligned}
& s(t)=x(t) u(t) \\
& \dot{x}=\frac{1-x(t)}{t_{D}}-u(t) x(t) \delta\left(t-t_{\text {spike }}\right) \\
& \dot{u}=\frac{U-u(t)}{t_{F}}-U[1-u(t)] \delta\left(t-t_{\text {spike }}\right)
\end{aligned}
$$


246 Here $\delta$ is the Dirac function and the state variables $x(t)$ and $u(t)$ have baseline levels of 1 247 and $U$ respectively. Parameters $t_{D}$ and $t_{F}$ are the depression and facilitating times which 248 govern how fast $x(t)$ and $u(t)$ return to baseline. Excitatory synapses' STP is modeled 249 with $U=0.5, t_{D}=800$ and $t_{F}=1000$ while inhibitory synapses have $U=0.2, t_{D}=700$ 250 and $t_{F}=20$. A small subgroup of excitatory neurons is stimulated by pre-synaptic spikes 251 distributed randomly in time without STP or STDP.

252 At each column, LPF is obtained assuming dendritic AMPA currents as a good indicator of 253 this activity by the average AMPA conductance in all afferent excitatory synapses (Seth, 254 Chorley \& Barnett, 2013).

255 In this work we simulated two columns of 5k randomly distributed Izhikevich neurons 256 (80\% excitatory, 20\% inhibitory) with STDP and STP between neurons of the same column 257 and without both learning strategies in inter-column connections. $5 \%$ of these excitatory 258 neurons are stimulated by pre-synaptic spikes distributed randomly in time without STP or 259 STDP. Instead of a single parameter to model the synaptic strength between columns, like 260 the coupling coefficients or the inter-variable AR coefficient seen in the previous models, 261 here it is possible to model three parameters related to inter-column connection strength.

262 These are the percentage of neurons in one column that project to another column $p_{\text {projection }}$, 263 the percentage of connections each branch does in the target column $p_{\text {branch }}$ and the 264 synaptic strength of the individual connections $k_{\text {unit }}$. Simulations produced $60 \mathrm{~s}$ of data (the 265 first $20 \mathrm{~s}$ were discarded to remove transient effects) with causal influence from column 1 266 to column 2. In this case, as three parameters define the coupling strength, the simulations 267 were done for each one individually: for varying $p_{\text {projection }}\left(p_{\text {projection }}=[0\right.$ to 1 in steps of $\left.2680.2], p_{\text {branch }}=0.05, k_{\text {unit }}=1, d_{21}=20 \mathrm{~ms}\right)$, for varying $p_{\text {branch }}\left(p_{\text {projection }}=0.2, p_{\text {branch }}=\right.$ $\left.269[0.01,0.05,0.1,0.3,0.6,1], k_{\text {unit }}=1, d_{21}=20 \mathrm{~ms}\right)$ and for varying $k_{\text {unit }}\left(p_{\text {projection }}=0.2\right.$, $\left.270 p_{\text {branch }}=0.05, k_{\text {unit }}=[0.01,0.05,0.1,0.3,0.6,1], d_{21}=20 \mathrm{~ms}\right)$. In conformity with the

271 previous generative models, simulations for different interaction delays were also

272 performed $d_{21}\left(p_{\text {projection }}=0.2, p_{\text {branch }}=0.05, k_{\text {unit }}=1, d_{21}=[4 \mathrm{~ms}\right.$ and 20 to $100 \mathrm{~ms}$ in 
273 steps of $20 \mathrm{~ms}$ ]). The spiking units are ate rest in their initial state. Each $60 \mathrm{~s}$ of data

274 required $\sim 52 \mathrm{~s}$ of simulation time.

275 2.4. EEG forward modeling

276 EEG is simulated with a simple forward model that relates the LFP as the activity of a 277 current dipole to the surface potential measured at the scalp. This relation is obtained by 278 representing the head as a multilayer surface, with each layer having constant isotropic 279 conductivity. Although there are realistically shaped multilayer head models like the finite280 element method or the boundary element method (Mosher, Leahy \& Lewis, 1999; Fuchs et 281 al., 2002; Darvas et al., 2004), these are more important in the EEG inverse problem 282 solution as they mitigate the distortion produced by simpler models (Ermer \& Mosher, 283 2001). As the scope of this work is to simulate a generic EEG signal we can adopt a simpler 284 model of the skull like the three layer sphere with isotropic conductivities (Berg \& Scherg, 285 1994) where the problem of volume conduction is observed. Figure 2 depicts a typical three 286 layer model with an electrode placed in the scalp with radius $\boldsymbol{r}$, an intracranial current 287 dipole with radius $\boldsymbol{r}_{\boldsymbol{q}}$ and moment $\boldsymbol{q}$ and respective angles. This experiment uses a setup 288 with two dipoles placed beneath the three layers with $8 \mathrm{~cm}$ outer radius $\left(\sigma_{\text {brain }}=0.33 \mathrm{~S} / \mathrm{m}\right.$ $\left.r_{\text {brain }}=7.04 \mathrm{~cm}, \sigma_{\text {skull }}=0.0042 \mathrm{~S} / \mathrm{m}, r_{\text {skull }}=7.44 \mathrm{~cm}, \sigma_{\text {scalp }}=0.33 \mathrm{~S} / \mathrm{m}, r_{\text {scalp }}=8 \mathrm{~cm}\right)$

290 spaced by $2 \mathrm{~cm}$ and two electrodes are placed in the scalp also spaced by $2 \mathrm{~cm}$.

\section{1 [INSERT FIGURE 2 HERE]}

292 The solution $v^{l}\left(\boldsymbol{r} ; \boldsymbol{r}_{\boldsymbol{q}} ; \boldsymbol{q}\right)$ at radius $\boldsymbol{r}$ for the simplest case of a single spherical layer head 293 model for a current dipole with moment $\boldsymbol{q}$ at radius location $\boldsymbol{r}_{\boldsymbol{q}}$ can be obtained by the sum 294 of the radial $v_{r}^{l}\left(\boldsymbol{r} ; \boldsymbol{r}_{\boldsymbol{q}} ; \boldsymbol{q}\right)$ and tangential $v_{t}^{l}\left(\boldsymbol{r} ; \boldsymbol{r}_{\boldsymbol{q}} ; \boldsymbol{q}\right)$ potentials:

$$
\begin{aligned}
& v_{r}^{I}\left(\boldsymbol{r} ; \boldsymbol{r}_{\boldsymbol{q}} ; \boldsymbol{q}\right)=\left(\frac{q \cos \alpha}{4 \pi \sigma}\right)\left(\frac{2\left(r \cos \gamma-r_{q}\right)}{d^{3}}+\frac{1}{r_{q} d}-\frac{1}{r r_{q}}\right) \\
& v_{t}^{I}\left(\boldsymbol{r} ; \boldsymbol{r}_{q} ; \boldsymbol{q}\right)=\left(\frac{q \sin \alpha}{4 \pi \sigma}\right) \cos \beta \sin \gamma\left(\frac{2 r}{d^{3}}+\frac{d+r}{r d\left(r-r_{q} \cos \gamma+d\right)}\right)
\end{aligned}
$$


295 Here, $\alpha$ is the angle the dipole expresses in relation to its location vector $\boldsymbol{r}_{q}$, as can be seen 296 in Figure 2, $\sigma$ is the conductivity of the shell, $d$ is the value of the direct distance between $297 \boldsymbol{r}_{q}$ and $\boldsymbol{r}$, and $\beta$ is the angle between the plane formed by $\boldsymbol{r}_{q}$ and $\boldsymbol{q}$, and the plane formed 298 by $\boldsymbol{r}_{q}$ and $\boldsymbol{r}$.

299 Following (Berg \& Scherg, 1994; Zhang, 1995) a three layer model can be approximated 300 with good accuracy by single layer spheres with the approximation $v^{3}\left(\boldsymbol{r} ; \boldsymbol{r}_{q} ; \boldsymbol{q}\right) \cong \mathrm{v}^{1}\left(\boldsymbol{r} ; \mu_{1} \boldsymbol{r}_{\boldsymbol{q}} ; \lambda_{1} \boldsymbol{q}\right)+\mathrm{v}^{1}\left(\boldsymbol{r} ; \mu_{2} \boldsymbol{r}_{\boldsymbol{q}} ; \lambda_{2} \boldsymbol{q}\right)+\mathrm{v}^{1}\left(\boldsymbol{r} ; \mu_{3} \boldsymbol{r}_{q} ; \lambda_{3} \boldsymbol{q}\right)$. The $\mu$ and $\lambda$ are the "Berg parameters" and are used to create three new dipoles with locations consisting in scaling $\boldsymbol{r}_{q}$ by $\mu$ in its radial direction and scaling the moment $\boldsymbol{q}$ by $\lambda$. The approximation $v^{3}\left(\boldsymbol{r} ; \boldsymbol{r}_{q} ; \boldsymbol{q}\right)$

304 is computed for each electrode and dipole present in the simulation. Noise is added to the 305 resulting time-series.

306 Details about the computation of the "Berg parameters" can be found in (Zhang, 1995) and 307 other methods for EEG forward models in (Mosher, Leahy \& Lewis, 1999; Ermer \& 308 Mosher, 2001; Darvas et al., 2004). The Brainstorm application offers several methods for 309 the EEG forward model among others (Tadel et al., 2011).

310 For our simulations, each generated LFP was fed to an EEG forward model with two radial

311 dipoles spaced by $2 \mathrm{~cm}$ placed beneath the three layers with $8 \mathrm{~cm}$ outer radius ( $\sigma_{\text {brain }}=0.33$

$312 \mathrm{~S} / \mathrm{m} r_{\text {brain }}=7.04 \mathrm{~cm}, \sigma_{\text {skull }}=0.0042 \mathrm{~S} / \mathrm{m}, r_{\text {skull }}=7.44 \mathrm{~cm}, \sigma_{\text {scalp }}=0.33 \mathrm{~S} / \mathrm{m}, r_{\text {scalp }}=8 \mathrm{~cm}$ )

313 and two recording electrodes placed in the scalp also spaced by $2 \mathrm{~cm}$. White Gaussian noise 314 was added in order for a linear SNR of 10.

316 The BOLD signal time-series is the result of a series of neuronal and vascular events that 317 produce a measurable change in the blood hemoglobin concentration. It is therefore an 318 indirect and noisy observation of the neuronal activity as during neuronal activation local 319 vessels are dilated to increase the blood flow and with it, oxygen and glucose delivery. The 320 increased metabolism results in a localized increase in the conversion of oxygenated 321 hemoglobin to deoxygenated hemoglobin and BOLD fMRI uses the latter as the contrast 
322 agent (Ogawa \& Lee, 1990). This activity can peak four seconds after the neuronal event

323 onset though this value varies within and between subjects (Handwerker, Ollinger \&

324 D'Esposito, 2004). Hence, the location, dynamics and magnitude of the BOLD signal's

325 activity are vastly influenced by the local vascular bed. This, combined with the fact that

326 fMRI scanners sample an entire volume with TR in the time scale of a second, raised the

327 question if directed functional connectivity, which aims at detecting temporal precedence

328 between neuronal events in the order of tens to hundreds of milliseconds (Ringo et al.,

329 1994; Formisano et al., 2002; de Pasquale et al., 2010), can offer accurate measures from

330 BOLD signals. The simulation of BOLD signals was important to answer this question by

331 allowing experimental control over neuronal and hemodynamic parameters and this has

332 been achieved mainly by convolution with a canonical hemodynamic response function

333 (HRF) or by dynamic modeling of the vascular activity with the extended Balloon-

334 Windkessel (BW) model (Friston et al., 2000).

335 The first approach started being used with one gamma function for the HRF convolution

336 kernel (Goebel, 2003; Roebroeck, Formisano \& Goebel, 2005) with the purpose of

337 investigating the effect of filtering, down-sampling and noise in GC estimation. Following

338 simulation studies (Deshpande, Sathian \& Hu, 2009; Schippers, Renken \& Keysers, 2011;

339 Seth, Chorley \& Barnett, 2013; Rodrigues \& Andrade, 2014) started using a dual-gamma

340 function as used in SPM software (Friston, K., Holmes, A., Ashburner, 1999) with

341 parameters as time to peak, time to undershoot, onset time, dispersion of response,

342 dispersion of undershoot and their ratio following the distributions found in (Handwerker,

343 Ollinger \& D'Esposito, 2004) to study the effects of down-sampling, noise and HRF

344 variability. Compared to the convolution approach, the BW model is more biophysically

345 interpretable and can also present nonlinear neuro-vascular couplings although when used

346 in simulation studies there were no considerable differences in GC estimates between both

347 (Smith et al., 2010; Seth, Chorley \& Barnett, 2013).

348 In this work, both approaches were used with the default parameters offered by SPM12 in

349 the functions spm_hdm_priors for the BW model and spm_hrf for the canonical HRF, with

350 a 0.5 TR and a linear SNR of 10. However, in these simulations, new LFPs had to be

351 created for all generative models with different interaction delays, duration and 
352 connectivity frequency. Interaction delays are increased to $200 \mathrm{~ms}$, in order to counteract 353 the reduction in sensitivity due to the low sampling period of typical fMRI TRs and low 354 SNR (Deshpande, Sathian \& Hu, 2009), and the LFP length had to be increased due to the 355 low sampling rate hence, 300 seconds were simulated. For the generative models where it is 356 possible to control the frequency where the causal influence is exerted these were set, from $35733 \mathrm{~Hz}$ in the previous analysis, to $0.1 \mathrm{~Hz}$ in the Kuramoto oscillators and $<1 \mathrm{~Hz}$ for the 358 MVAR modeling.

3592.6 Spectral Granger causality

360 We use the standard Geweke's spectral decomposition for GC (GGC) (Geweke, 1982) to 361 infer causality between synthetic time-series. Unlike other spectral directed functional 362 connectivity metrics such as PDC or DTF, GGC is not bounded between 0 and 1 which is 363 useful in this study to see how the increase in the connection strength between variables 364 effects the absolute value of causality across the different simulation methods. GGC 365 decomposes the GC index (GCI) (Granger, 1969) into frequency components additively, 366 meaning that, the sum of all the frequency components from zero to the Nyquist frequency 367 result in the GCI. For bivariate time-series, which is the case in this study, GGC can be 368 computed from a MVAR model parameters by:

$$
\mathrm{F}_{\mathrm{j} \rightarrow \mathrm{i}}(f)=\ln \frac{S_{i i}(f)}{S_{i i}(f)-\left(\Sigma_{i j}-\frac{\Sigma_{i j}^{2}}{\sum_{i i}}\right)\left|H_{i j}(f)\right|^{2}} j, i \in\{1,2\}
$$

369 Here $\Sigma$ is the covariance matrix of the model's errors, $H$ is the transfer matrix, and $S$ is

370 the spectral matrix. The MVAR model order can be estimated with the Bayesian

371 information criterion (BIC) (Schwarz, 1978) or with the Akaike information criterion (AIC)

372 (Akaike, 1974).

373 For multivariate time-series refer to (Chen, Bressler \& Ding, 2006) for more details.

374 3. Results

375 This section presents the results of applying (11) to bivariate time-series simulated with the 376 generative and forward models introduced in Figure 1 and in the previous sections with 377 varying interaction strength and delay. This analysis aims at finding whether these changes 
378 in the generative modeling are captured by standard causality estimation by analyzing how

379 GGC's difference of influence (DOI) $\left(F_{j \rightarrow i}-F_{i \rightarrow j}\right.$ when $F_{j \rightarrow i}$ is the true causal direction)

380 varies with the modeled interaction strength and how GGC's DOI and estimated MVAR

381 model order estimation change with increasing interaction delays. An increase in empirical

382 model order from BIC or AIC suggests that the interaction delays introduced in the

383 generative model are detected in the resulting time-series.

384 3.1. LFP

385 LFPs represent the local activity of neuronal populations without forward modeling hence, 386 without the confounding effects expected from fMRI BOLD signals or EEG data and, the 387 only randomness is due to the stochastic parameters from each model. This way, this 388 analysis tests each generative model's capability to produce synthetic datasets with 389 detectable causal effects.

$390 \quad$ 3.1.1 AR modeling

391 Results for GGC with varying modeled causality and interaction delays can be seen in 392 Figures 3A and 4A respectively and the model order estimated with BIC and AIC for the 393 different interaction delays are shown in Figure 5A.

394 The estimated GGC DOI present in Figure 3A shows, as expected, causal influence at 33 $395 \mathrm{~Hz}$ with the same absolute value from what was modeled. Changing the interaction delay 396 from 4 to 100 ms doesn't affect the estimated causality, as can be seen in Figure 4A, and 397 both BIC and AIC model orders increase linearly with the increase in interaction delay 398 (Figure 5A). Also, these model orders correspond exactly to the neuronal delay: with a 399 sampling rate of $250 \mathrm{~Hz}$; the neuronal delays in $d_{21}$ correspond to [1, 5 to 25 in steps of 5] 400 lagged observations which equal the model orders suggested by BIC and AIC (except for 401 the neuronal delay of $4 \mathrm{~ms}$ ).

402 3.1.2. Time-delayed coupled $K I_{e, i}$ sets

403 Figures 3B and 4B respectively show the estimated GGC for varying coupling and 404 interaction delay and Figure 5B the respective model orders estimated with BIC and AIC. 
405 Figure 3B shows that there are two frequency bands where connectivity is detected, around $40620 \mathrm{~Hz}$ and around $60 \mathrm{~Hz}$. These causal influences are more expressive for strong couplings $407(>1)$ and are inexistent at weak couplings below 0.5. When the interaction delay changes, 408 connectivity also changes its frequency (Figure 4B). This occurs as different delays change 409 the oscillatory behavior of these neuronal populations and also because the MVAR model 410 order also changes which influences the AR spectrum. In Figure 5B it is possible to see that 411 both BIC and AIC are sensitive to the increase in the interaction delay and overestimate the 412 true model order by approximately 2 lagged observations.

413 3.1.3. Phase-delayed coupled Kuramoto oscillators

414 Figures $3 \mathrm{C}$ and $4 \mathrm{C}$ respectively show the estimated GGC for varying coupling and 415 interaction delay and Figure 5C the respective model orders estimated with BIC and AIC

416 GGC only detects causal relations with positive DOI at $33 \mathrm{~Hz}$ as can be seen in Figure 3C.

417 However, these only present expressive values on strong couplings (>3). Different 418 interaction delays do not affect the GGC intensity or frequency distribution (Figure 4C)

419 although the model order suggested by BIC and AIC is not linearly related to the

420 interaction delay (Figure 5C) and is overestimated.

$421 \quad 3.1 .4$ Izhikevich columns

422 GGC results for varying $p_{\text {projection }}$ can be seen in Figure 3D, results for varying $p_{\text {branch }}$ can 423 be seen in Figure 3E and results for varying $k_{\text {unit }}$ can be seen in Figure 3F. GGC results for 424 simulations with varying are shown in Figure 4D and the respective model orders estimated 425 with BIC and AIC in Figure 5D.

426 From Figures 3D, 3E, 3F it is possible to see that GGC detects connectivity below $20 \mathrm{~Hz}$ 427 and that a linear increase only occurs with $k_{\text {unit }}$. Interaction delays change the connectivity 428 frequency distribution (Figure 4D) as different delays change the oscillatory behavior of the 429 neuronal populations and different MVAR model order produce different AR spectrums. 430 Increasing the interaction delay produces a linear increase in model orders estimated with 431 BIC although these are slightly overestimated (Figure 5D). 
[INSERT FIGURE 4 HERE]

434

435

436

437

438

439

440

441

442

443

444

445

446

447

448

449

450

451

452

453

454

455

456

457

458

\section{[INSERT FIGURE 5 HERE]}

\subsection{EEG forward modeling}

Figure 6 shows the same experiments as Figure 3 only this time, the time-series is the EEG recorded at the scalp electrodes. It is possible to see that, except for the Izhikevich columns, GGC amplitude is reduced.

[INSERT FIGURE 6 HERE]

EEG forward modeling does not affect the detection of interaction delays as can be seen in Figure 7. However, for the $K I_{e, i}$ sets, causality around the $20 \mathrm{~Hz}$ dissipates and causality at $60 \mathrm{~Hz}$ is maintained. At some delays $(0.4$ and $0.8 \mathrm{~ms})$ the EEG from the Kuramoto oscillators (Figure 7C) shows GGC values similar to the LFPs.

\section{[INSERT FIGURE 7 HERE]}

Overall model orders are overestimated for EEGs from every generative models. In the EEG from MVAR modeling and $K I_{e, i}$ sets (Figures $8 \mathrm{~A}$ and $8 \mathrm{~B}$ respectively) this is more pronounced in the lower interaction delays and in the EEG from the Izhikevich columns (Figure 8D) the opposite seems to occur. The overestimation problems in the LFPs from the Kuramoto oscillators are more pronounced after EEG forward modeling (Figure 8C).

\section{[INSERT FIGURE 8 HERE]}

\subsection{BOLD forward modeling}

The results for both the convolution with canonical HRF and the extended BW model are shown in Figures 9 and 10.

The results in Figure 9 show that GGC is greatly reduced after BOLD forward modeling regardless of the generative or forward models. In BOLD signals generated with MVAR modeling causal effects are more visible after a modeled causality of 2.5 (Figure 9A) although these remain around 0.5 . The same values are achieved by $K I_{e, i}$ sets and Kuramoto oscillators after a coupling of 1 and 10 respectively. The Izhikevich columns only achieved 
459 these values for $p_{\text {projection }}>0.4, p_{\text {branch }}>0.6$ or $k_{\text {unit }}>0.3$. Both the canonical HRF and the 460 extended BW show similar results.

461 [INSERT FIGURE 9 HERE]

462 By keeping the coupling strengths at the maximum values in Figure 9 and varying the 463 interaction delay from $100 \mathrm{~ms}$ to $300 \mathrm{~ms}$ the results in Figure 10 suggest that all generative 464 and forward models benefit from higher delays. With MVAR modeling, $150 \mathrm{~ms}$ is the 465 value when causality is detectable (Figure 10A) whereas for the remaining generative 466 models this value is at $200 \mathrm{~ms}$. Again, there are no relevant differences between canonical 467 HRF and extended BW models.

468 [INSERT FIGURE 10 HERE]

469 4. Discussion

470 This study explores four generative models that represent distinct methodologies:

471 multivariate MVAR modeling, neural mass models and spiking neuron populations.

472 Although most of these models have been used previously in simulation studies that aim at 473 benchmarking connectivity metrics, their capability to reflect causal interactions in their 474 generated neuronal time-series had not been compared yet. Also, these neuronal time-series 475 are not limited to the LFPs, BOLD signals and EEG covered by this work but these are the 476 most widely used in connectivity studies.

477 Concerning the generative models in this work, both MVAR modeling and Kuramoto 478 oscillators offer the possibility to directly specify the frequency of their oscillatory activity 479 and therefore the frequency where connectivity occurs whereas in $K I_{e, i}$ sets and Izhikevich 480 columns this is not possible, at least directly. On the other hand, $K I_{e, i}$ sets and Izhikevich 481 columns are neurophysiologically plausible as they offer the possibility to modulate 482 different types of excitatory and inhibitory neurons. This suggests two different uses for 483 these subgroups of generative models. MVAR modeling and Kuramoto oscillators, as these 484 allow to manipulate the strength and frequency of causal relationships (or even the 485 theoretical GGC in the MVAR modeling), seem more adequate for initial testing of directed 486 functional connectivity metrics (Baccalá \& Sameshima, 2001), studying the effect of post 487 processing or other transformations in data prior to causality inference (Barnett \& Seth, 
2011) or to compare different metrics performance in an extended benchmark (Rodrigues \&

489 Andrade, 2014). Due to their superior neurophysiological realism $K I_{e, i}$ sets and Izhikevich

490 columns are more useful to inquire about the effectiveness of certain connectivity metrics

491 for data recorded in specific brain locations with known dynamics (Friston et al., 2014). For

492 example, in (Freeman, 1987) coupled $K I$ sets are used to simulate chaotic EEG emanating

493 from the olfactory system and in (Richert et al., 2011) Izhikevich columns are used to

494 simulate a large-scale model of cortical areas V1, V4, and middle temporal (MT) with

495 color, orientation and motion selectivity.

496 All generative models are able to produce LFPs with detectable causal relations and these

497 results show the relation between experimental parameters and simulated causality. Only

498 MVAR modeling allows for a direct specification of causality by solving the analytical

499 equations for GGC applied to the MVAR coefficients. Concerning the neural mass models,

500 the $K I_{e, i}$ sets show causal effects with lower coupling strength than the Kuramoto oscillators

501 as the first start having causal relations with $k_{21}=0.7$ while the second required values of

$502 k>3$ for identifiable causality. In the Izhikevich columns the percentage of projecting

503 neurons from the source column $p_{\text {projection }}$ is the variable with least influence in the

504 observed GGC, except for when it is zero. Increases in both the percentage of target

505 connections per projection $p_{\text {branch }}$ and the synaptic strength $k_{\text {unit }}$ lead to increases in the

506 observed GGC. Although it wasn't tested in this work it is possible that these values change

507 for different number of neurons per column. In all generative models the modulation of the

508 interaction delay is possible without loss of causal relations although the

509 neurophysiologically realistic models show different frequency spectrums for different

510 interaction delays. This neuronal delay is also detected by the BIC and AIC which suggest

511 higher model orders for higher interaction delays.

512 Forward models lead to a decrease in the absolute value of GGC, specially the forward

513 BOLD model where negative DOIs could be found in worst scenarios. EEG forward

514 modeling reduces the estimated GGC due to the added noise and to the volume conduction

515 effect that produces a "cross-talk" between the two neural populations and electrodes and

516 these effects would be more adverse if more neuronal populations were added inside the

517 three-shell sphere. Nevertheless, these results show that all generative models produce 
518 LFPs that, when treated as activity from radial dipoles, preserve the causal relations in the

519 resulting EEG. BOLD forward modeling is more detrimental to the preservation of causal

520 effects and this work did not model hemodynamic variability between brain regions

521 (Handwerker, Ollinger \& D’Esposito, 2004) which would further affect causality

522 preservation in BOLD signal time-series (Deshpande, Sathian \& Hu, 2009). Causality was

523 reduced approximately by a factor of 10 in all generative models and there were no concise

524 differences between HRF convolution and BW modeling except for the Kuramoto models

525 where the last leads to smaller values of causality.

526 The overview from Table 1 confirms our initial suggestions that AR models are suitable for

527 exhaustive benchmarks of causal measures to study their dependence on experimental

528 parameters and formulation due to their low computational load associated with versatility

529 and analytical solution. For benchmarks with fewer experimental parameters and increased

530 concern in emulating neuronal data from frequency specific synchronized populations the

531 Kuramoto oscillators offer the best compromise between versatility and computational

532 load. Finally, to study causality in specific known neuronal dynamics both the $K I_{e, i}$ sets or

533 the Izhikevich are the most appropriate thanks to their ability to realistically simulate varied

534 neuronal populations.

535 [INSERT TABLE 1 HERE]

536 5. Conclusion

537 This work presented and analyzed different modeling strategies to generate artificial

538 neuronal datasets for benchmarking purposes. LFPs are obtained by generative models and

539 can be used by forward models to produce other recordings of neuronal activity such as the

540 BOLD signal or EEG. All the analyzed models were able to transmit their causal structure (

$\left.541 \varphi_{21}(1), k_{21}, k, p_{\text {projection }}, p_{\text {branch }}, k_{\text {unit }}\right)$ into their generated data however with different

542 relations between these and the identified GGC. This study only covered bivariate models

543 but the same analysis could be performed with larger networks with large-scale fluctuations

544 (Cabral et al., 2011). This would be useful to identify the directed functional connectivity

545 metrics most appropriate to analyze large scale data such as fMRI BOLD signals from

546 resting state networks. 
547 Acknowledgments

548 We acknowledge the Portuguese Foundation for Science and Technology (FCT) for

549 financial support through Project PTDC/SAU-ENB/112294/2009 and PEst-

550 OE/SAU/UI0645/2014.

551 
Akaike H. 1974. A new look at the statistical model identification. Automatic Control, IEEE Transactions on 19:716-723.

Baccalá L a, Sameshima K. 2001. Partial directed coherence: a new concept in neural structure determination. Biological cybernetics 84:463-74.

Barnett L, Seth AK. 2011. Behaviour of Granger causality under filtering: theoretical invariance and practical application. Journal of neuroscience methods 201:404-19.

Berg P, Scherg M. 1994. A fast method for forward computation of multiple-shell spherical head models. Electroencephalography and Clinical Neurophysiology 90:58-64.

Blinowska KJ, Franaszczuk PJ. 1989. A Model of the Generation of Electrocortical Rhythms. In: Başar E, Bullock TH eds. Brain Dynamics. Berlin, Heidelberg: Springer Berlin Heidelberg, 192-201.

Börgers C, Kopell N. 2003. Synchronization in networks of excitatory and inhibitory neurons with sparse, random connectivity. Neural computation 15:509-38.

Cabral J, Hugues E, Sporns O, Deco G. 2011. Role of local network oscillations in restingstate functional connectivity. NeuroImage 57:130-9.

Chen Y, Bressler SL, Ding M. 2006. Frequency decomposition of conditional Granger causality and application to multivariate neural field potential data. Journal of neuroscience methods 150:228-37.

Darvas F, Pantazis D, Kucukaltun-Yildirim E, Leahy RM. 2004. Mapping human brain function with MEG and EEG: methods and validation. NeuroImage 23 Suppl 1:S28999.

Deco G, Jirsa VK, McIntosh AR. 2011. Emerging concepts for the dynamical organization of resting-state activity in the brain. Nature reviews. Neuroscience 12:43-56.

Deshpande G, Sathian K, Hu X. 2009. Effect of hemodynamic variability on Granger causality analysis of fMRI. NeuroImage 52:884-896.

Eichler M. 2006. On the evaluation of information flow in multivariate systems by the directed transfer function. Biological cybernetics 94:469-82.

Ermer J, Mosher J. 2001. Rapidly recomputable EEG forward models for realistic head shapes. Physics in Medicine and ... 46:1265-1281.

Formisano E, Linden DEJ, Salle F Di, Trojano L, Esposito F, Sack AT, Grossi D, Zanella FE, Goebel R, Goethe-universita KDJW et al. 2002. Tracking the Mind's Image in the Brain I : Time-Resolved fMRI during Visuospatial Mental Imagery. Neuron 35:185-194.

Freeman WJ. 1987. Simulation of chaotic EEG patterns with a dynamic model of the olfactory system. Biological Cybernetics 56:139-150.

Friston KJ, Bastos AM, Oswal A, van Wijk B, Richter C, Litvak V. 2014. Granger causality revisited. NeuroImage 101:796-808.

Friston KJ, Mechelli A, Turner R, Price CJ. 2000. Nonlinear responses in fMRI: the Balloon model, Volterra kernels, and other hemodynamics. NeuroImage 12:466-77.

Friston, K., Holmes, A., Ashburner J. 1999. Statistical Parametric Mapping (SPM).

Fuchs M, Kastner J, Wagner M, Hawes S, Ebersole JS. 2002. A standardized boundary element method volume conductor model. Clinical neurophysiology : official journal of the International Federation of Clinical Neurophysiology 113:702-12.

Geweke J. 1982. Measurement of linear dependence and feedback between multiple time series. Journal of the American Statistical Association 77:304-313. 
598

599

600

601

602

603

604

605

606

607

608

609

610

611

612

613

614

615

616

617

618

619

620

621

622

623

624

625

626

627

628

629

630

631

632

633

634

635

636

637

638

639

640

641

642

643
Goebel R. 2003. Investigating directed cortical interactions in time-resolved fMRI data using vector autoregressive modeling and Granger causality mapping. Magnetic Resonance Imaging 21:1251-1261.

Granger CWJ. 1969. Investigating Causal Relations by Econometric Models and Crossspectral Methods. Econometrica 37:424-438.

Handwerker D a, Ollinger JM, D'Esposito M. 2004. Variation of BOLD hemodynamic responses across subjects and brain regions and their effects on statistical analyses. Neurolmage 21:1639-51.

Izhikevich EM. 2003. Simple model of spiking neurons. IEEE transactions on neural networks / a publication of the IEEE Neural Networks Council 14:1569-72.

Izhikevich EM. 2004. Which model to use for cortical spiking neurons? IEEE transactions on neural networks / a publication of the IEEE Neural Networks Council 15:1063-70.

Jiru A. 2008. RELATIONSHIPS BETWEEN SPECTRAL PEAK FREQUENCIES OF A CAUSAL AR(P) PROCESS AND ARGUMENTS OF ROOTS OF THE ASSOCIATED AR POLYNOMIAL. San Jose State University.

Kaminski M, Blinowska KJ. 2014. Directed Transfer Function is not influenced by volume conduction-inexpedient pre-processing should be avoided. Frontiers in computational neuroscience 8:61.

Kamiński M, Ding M, Truccolo W a., Bressler SL. 2001. Evaluating causal relations in neural systems: Granger causality, directed transfer function and statistical assessment of significance. Biological Cybernetics 85:145-157.

Kloeden PE, Platen E. 1992. Numerical Solution of Stochastic Differential Equations. Berlin, Heidelberg: Springer Berlin Heidelberg.

Korzeniewska A, Mańczak M, Kamiński M, Blinowska KJ, Kasicki S. 2003. Determination of information flow direction among brain structures by a modified directed transfer function (dDTF) method. Journal of Neuroscience Methods 125:195-207.

Kuramoto Y. 1984. Chemical Oscillations, Waves, and Turbulence. Springer-Verlag, Berlin.

Londei A, D'Ausilio A, Basso D, Belardinelli MO. 2006. A new method for detecting causality in fMRI data of cognitive processing. Cognitive processing 7:42-52.

Mattia M, Del Giudice P. 2002. Population dynamics of interacting spiking neurons. Physical Review E 66:051917.

Mosher J, Leahy R, Lewis P. 1999. EEG and MEG: forward solutions for inverse methods. ... , IEEE Transactions on 46:245-259.

Ogawa S, Lee T. 1990. Brain magnetic resonance imaging with contrast dependent on blood oxygenation. Proceedings of the ... 87:9868-9872.

De Pasquale F, Della Penna S, Snyder AZ, Lewis C, Mantini D, Marzetti L, Belardinelli P, Ciancetta L, Pizzella V, Romani GL et al. 2010. Temporal dynamics of spontaneous MEG activity in brain networks. Proceedings of the National Academy of Sciences of the United States of America 107:6040-5.

Richert M, Nageswaran JM, Dutt N, Krichmar JL. 2011. An efficient simulation environment for modeling large-scale cortical processing. Frontiers in neuroinformatics 5:19.

Ringo JL, Don RW, Demeter S, Simard PY. 1994. Time Is of the Essence: A Conjecture that Hemispheric Specialization Arises from Interhemispheric Conduction Delay. Cereb. Cortex 4:331-343. 
Rodrigues J, Andrade A. 2014. Lag-based effective connectivity applied to fMRI: a simulation study highlighting dependence on experimental parameters and formulation. NeuroImage 89:358-77.

Roebroeck A, Formisano E, Goebel R. 2005. Mapping directed influence over the brain using Granger causality and fMRI. NeuroImage 25:230-42.

Sato JR, Fujita A, Cardoso EF, Thomaz CE, Brammer MJ, Amaro E. 2010. Analyzing the connectivity between regions of interest: an approach based on cluster Granger causality for fMRI data analysis. NeuroImage 52:1444-55.

Schippers MB, Renken R, Keysers C. 2011. The effect of intra- and inter-subject variability of hemodynamic responses on group level Granger causality analyses. NeuroImage 57:22-36.

Schwarz G. 1978. Estimating the dimension of a model. The annals of statistics 6:461-464. Seth AK. 2010. A MATLAB toolbox for Granger causal connectivity analysis. Journal of neuroscience methods 186:262-73.

Seth AK, Chorley P, Barnett LC. 2013. Granger causality analysis of fMRI BOLD signals is invariant to hemodynamic convolution but not downsampling. NeuroImage 65:540 555.

Smith SM, Miller KL, Salimi-Khorshidi G, Webster M, Beckmann CF, Nichols TE, Ramsey JD, Woolrich MW. 2010. Network Modelling Methods for FMRI. Neurolmage 54:875-891.

Tadel F, Baillet S, Mosher JC, Pantazis D, Leahy RM. 2011. Brainstorm: a user-friendly application for MEG/EEG analysis. Computational intelligence and neuroscience 2011:879716.

Valdes-Sosa P a, Roebroeck A, Daunizeau J, Friston K. 2011. Effective connectivity: Influence, causality and biophysical modeling. NeuroImage 58:339-361.

Zhang Z. 1995. A fast method to compute surface potentials generated by dipoles within multilayer anisotropic spheres. Physics in Medicine and Biology 40:335-349. 
Strategies used for synthetic neural data modeling.

LFPs are simulated by four distinct generative models and the resulting time-series can be used by EEG or BOLD forward models to produce the respective signals.

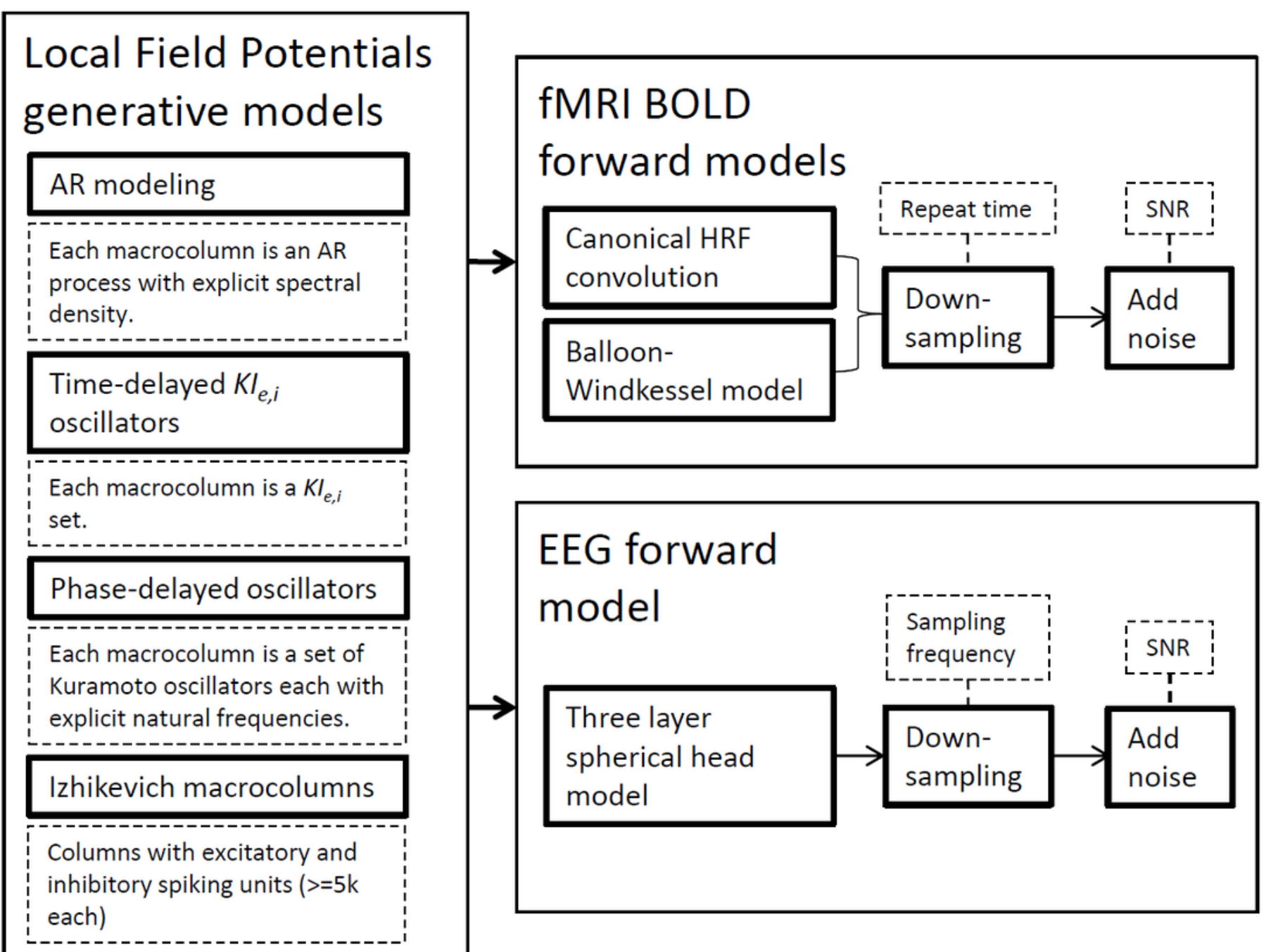


2

Three layer spherical head model:

for one current dipole with radius $\boldsymbol{r}_{\boldsymbol{q}}$ and moment $\boldsymbol{q}$ and scalp electrode with radius $\boldsymbol{r}$ oriented with angles $\alpha$ and $\gamma$ respectively. Adapted from (Mosher, Leahy \& Lewis, 1999).

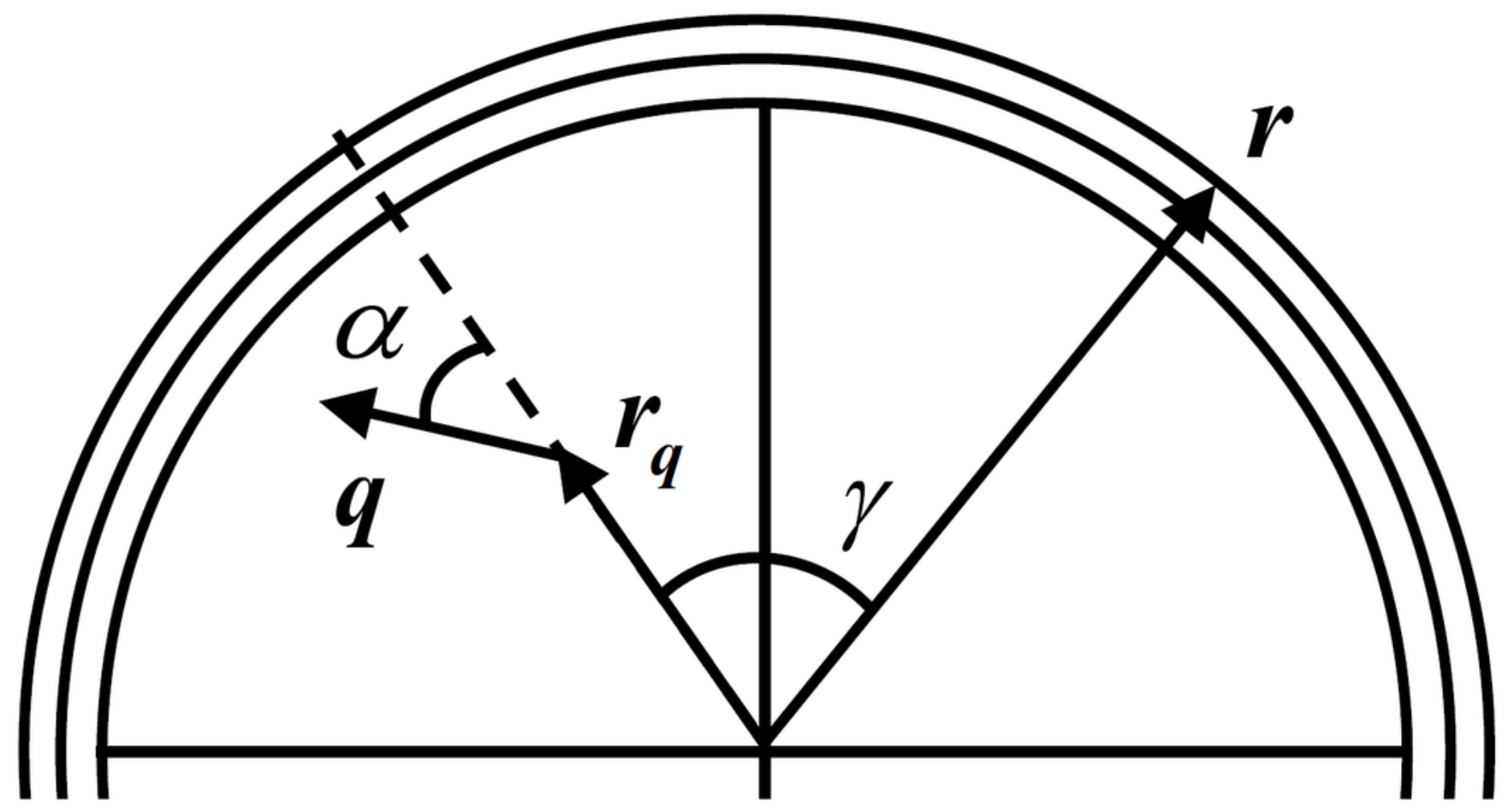


3

GGC DOI for the time-series simulated with increasing coupling strength for the generative models:

A) MVAR models, B) $K_{e, i}$ sets, C) Kuramoto oscillators, D, E and F) Izhikevich columns. 
A)

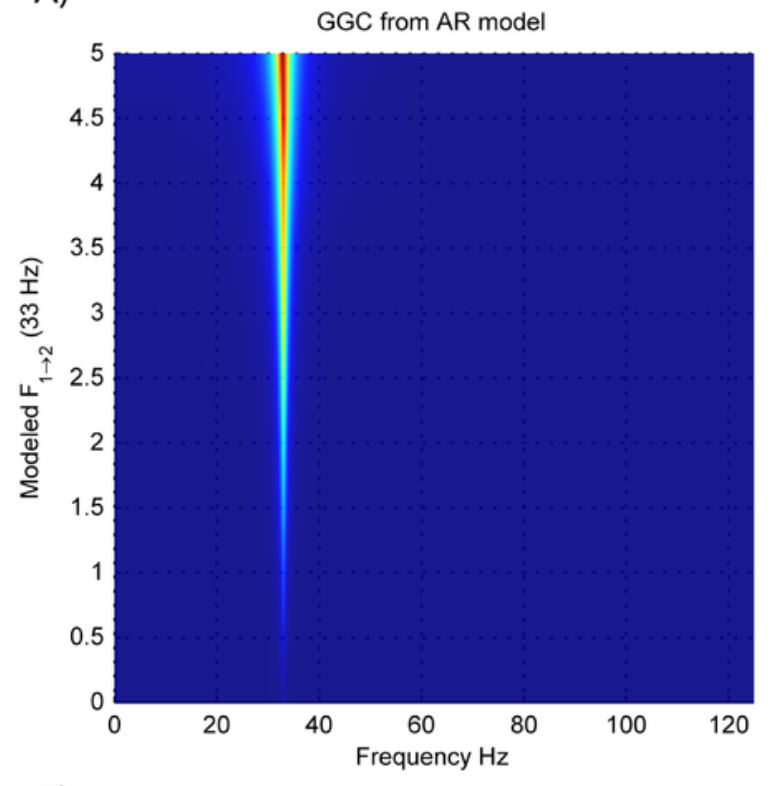

C)

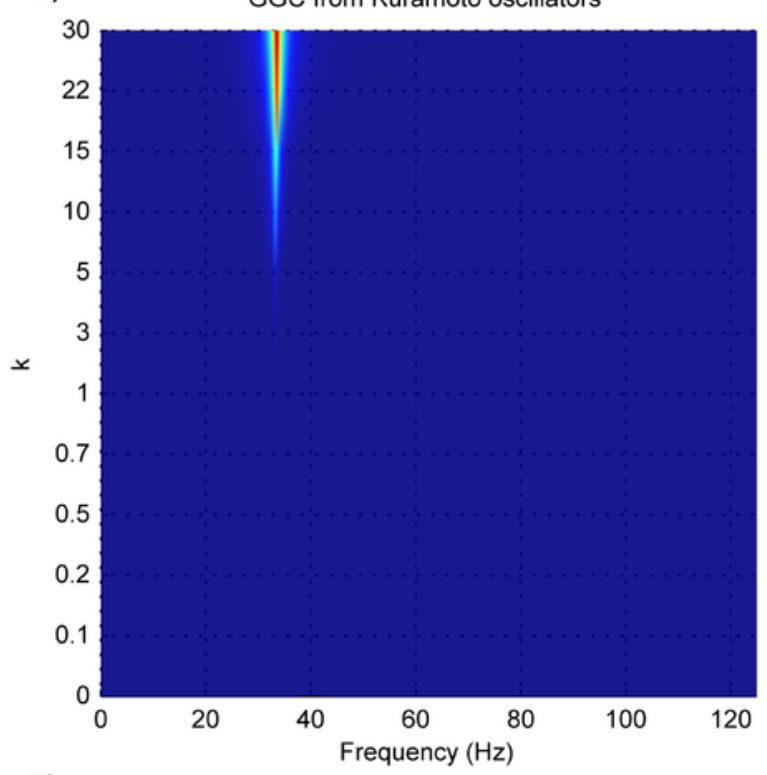

E)

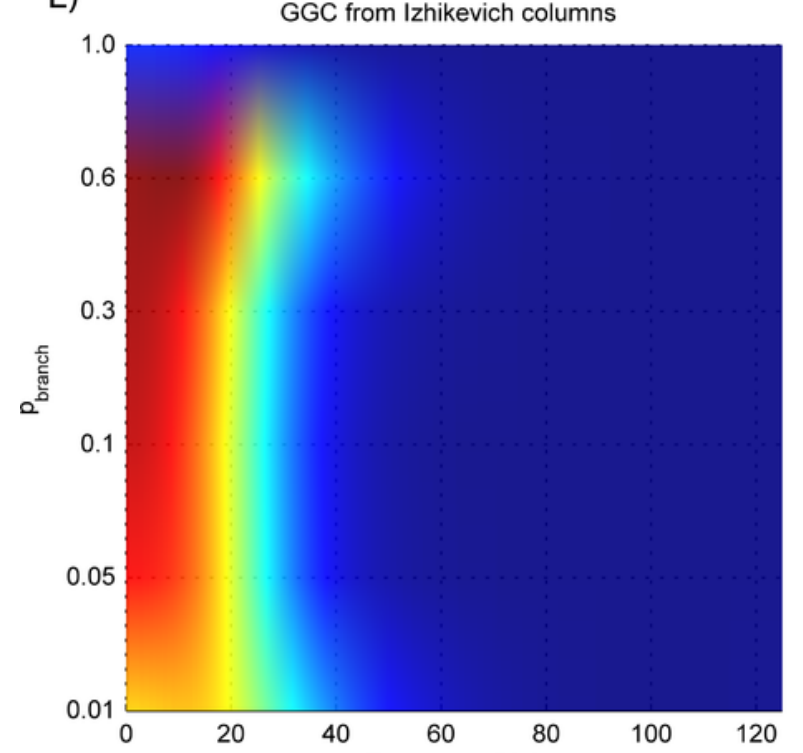

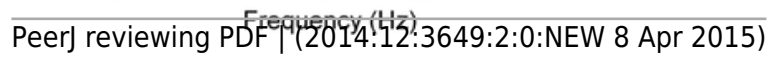

B)

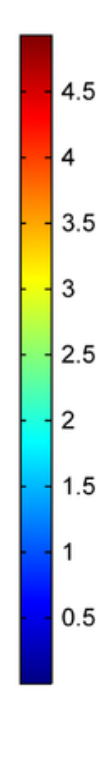

GGC from $\mathrm{KI}_{\text {ei }}$ sets

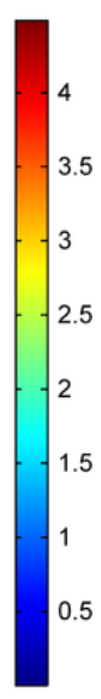

D)
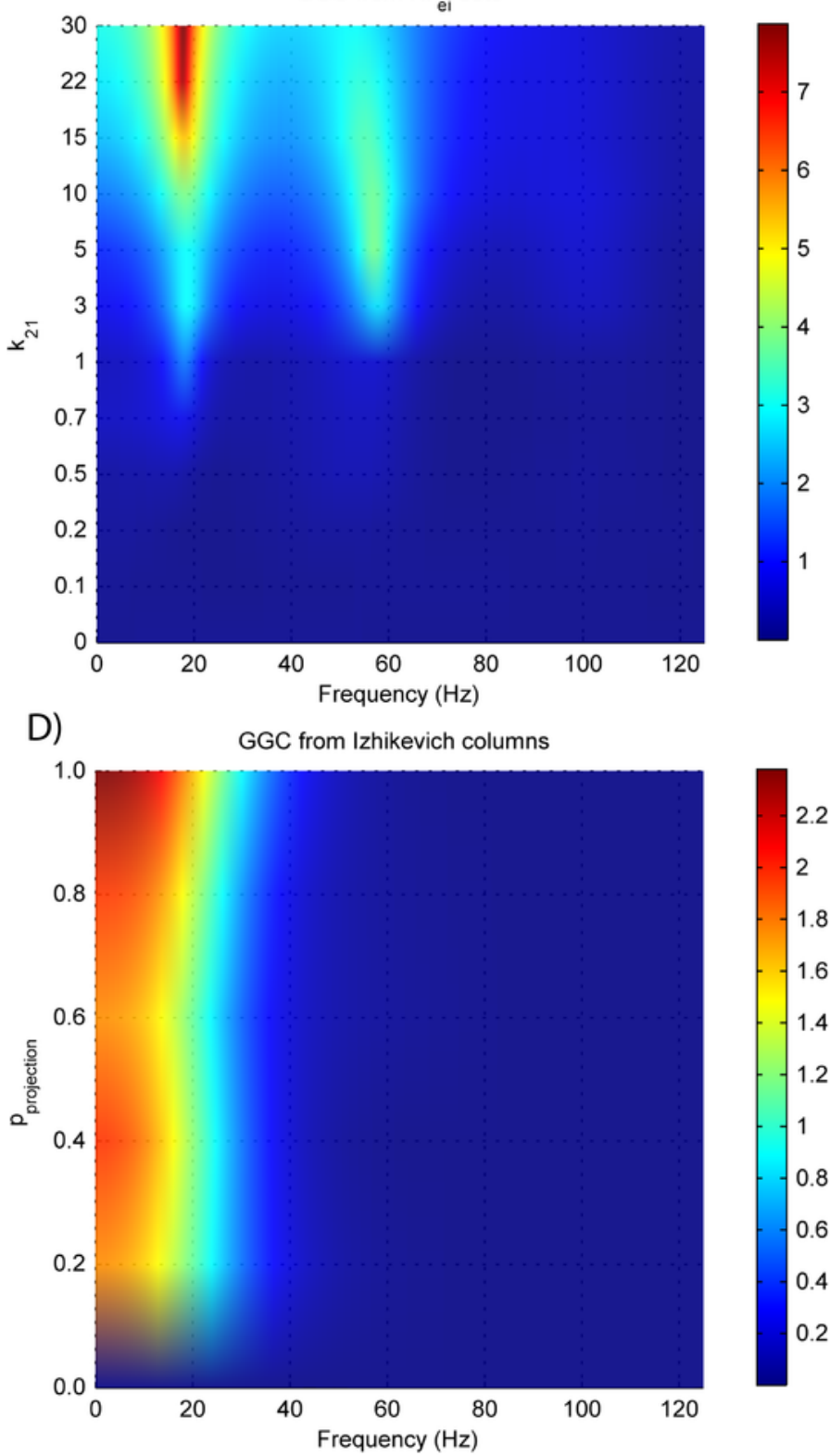

GGC from Izhikevich columns

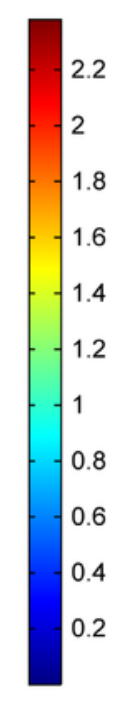

F)
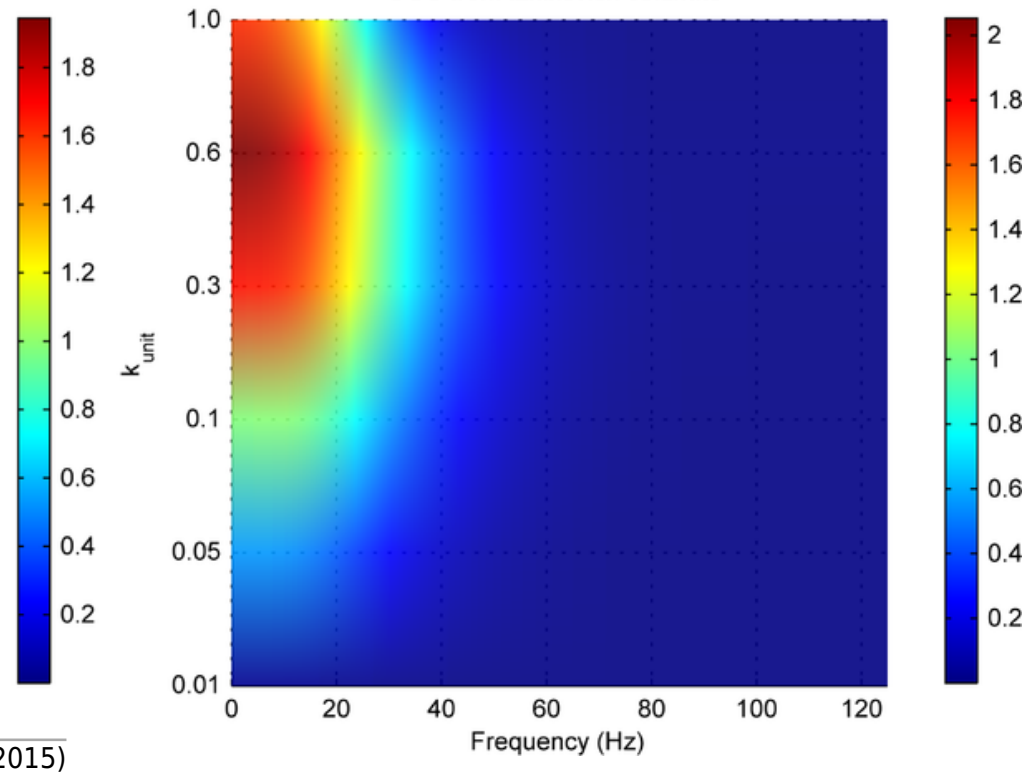
4

GGC DOI for the time-series simulated with increasing interaction delay for the generative models:

A) MVAR models, B) Kle,i sets, C) Kuramoto oscillators, D) Izhikevich columns.

A)

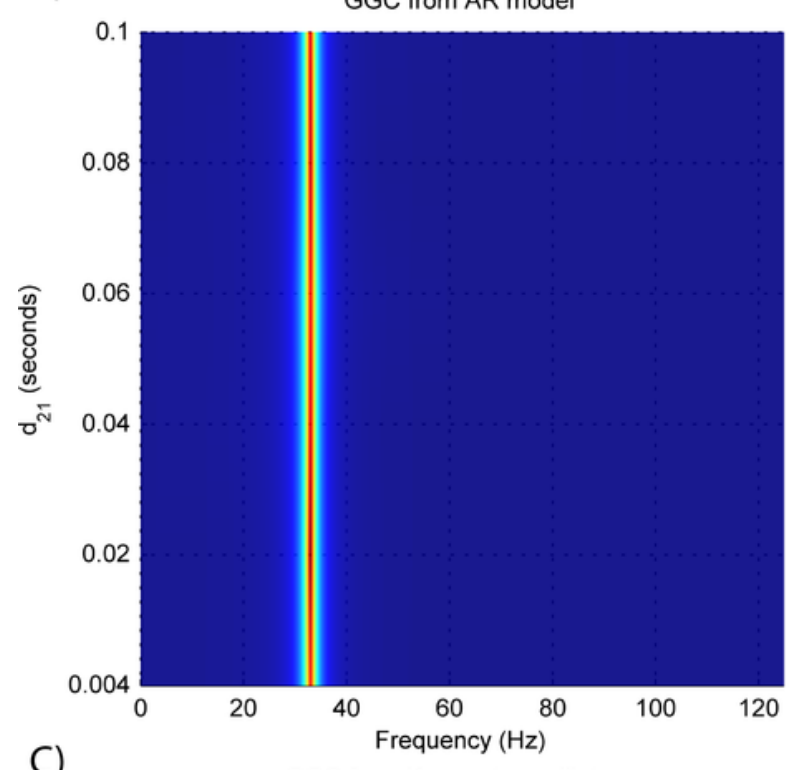

C)

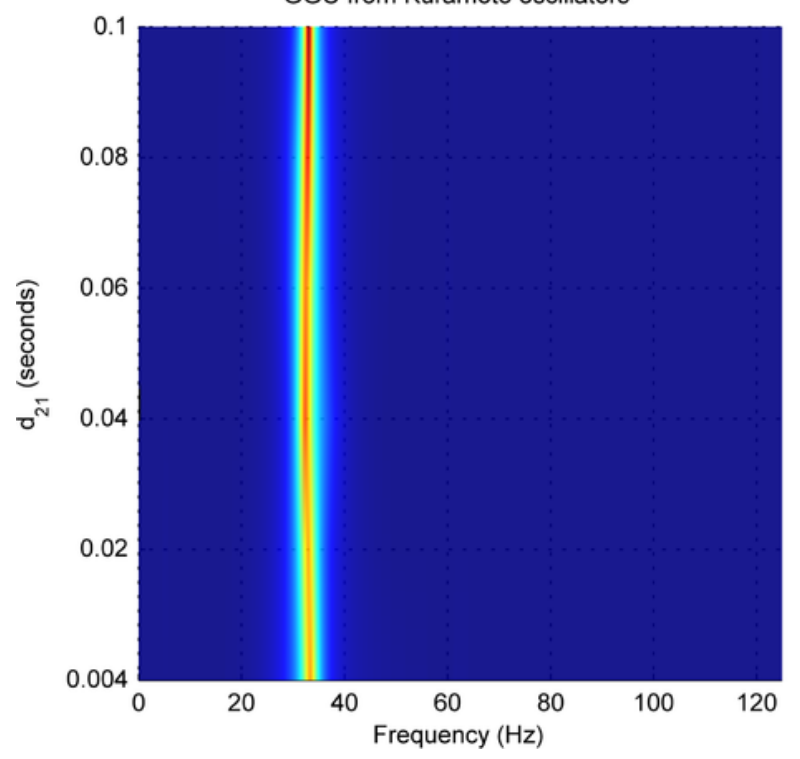

B)
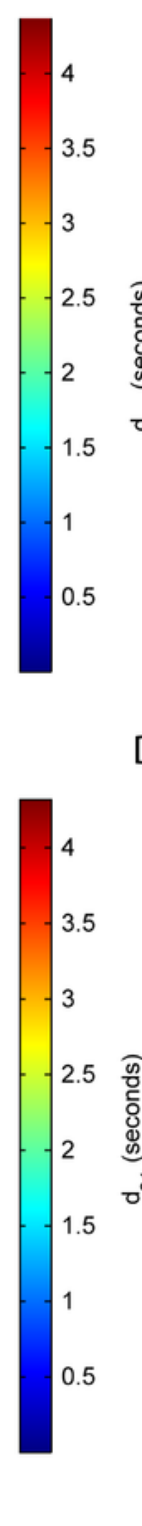

D)

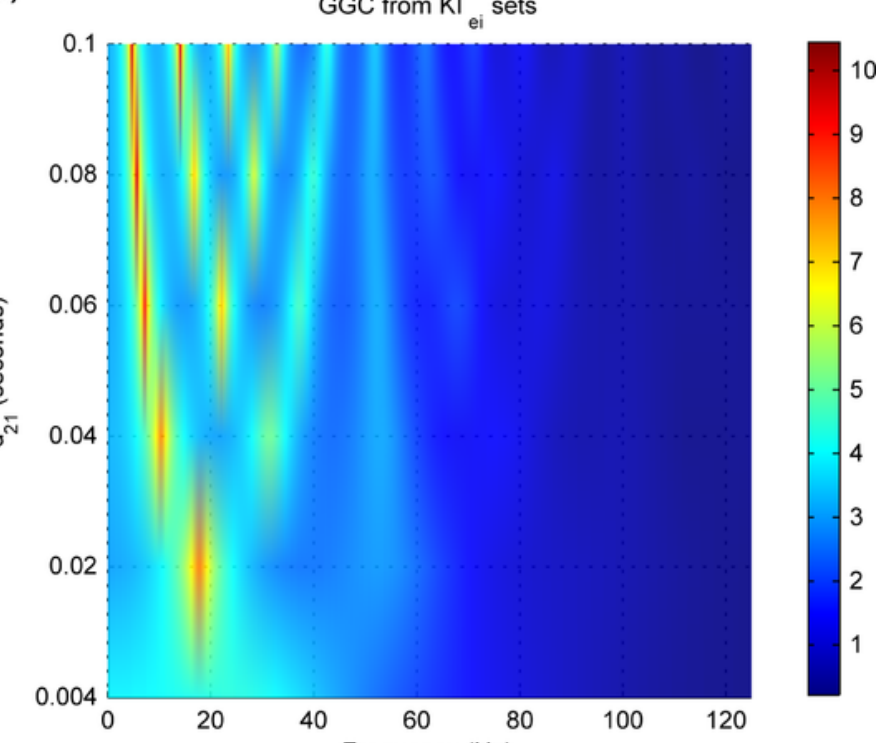
Frequency $(\mathrm{Hz})$ GGC from Izhikevich columns

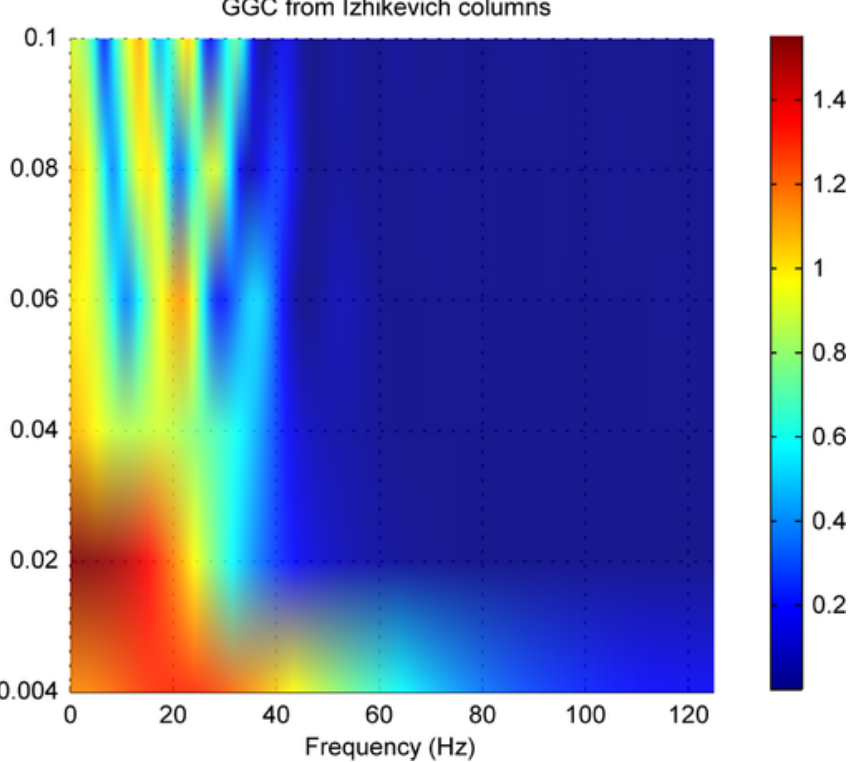




\section{5}

Model orders estimated with $\mathrm{BIC}$ and $\mathrm{AIC}$ for the time-series simulated with increasing interaction delay for the generative models:

A) MVAR models, B) $K l_{e, i}$ sets, C) Kuramoto oscillators, D) Izhikevich columns. The lagged observations for these interaction delays with $250 \mathrm{HZ}$ sampling rate are $[1,5,10,15,20,25]$.

A)

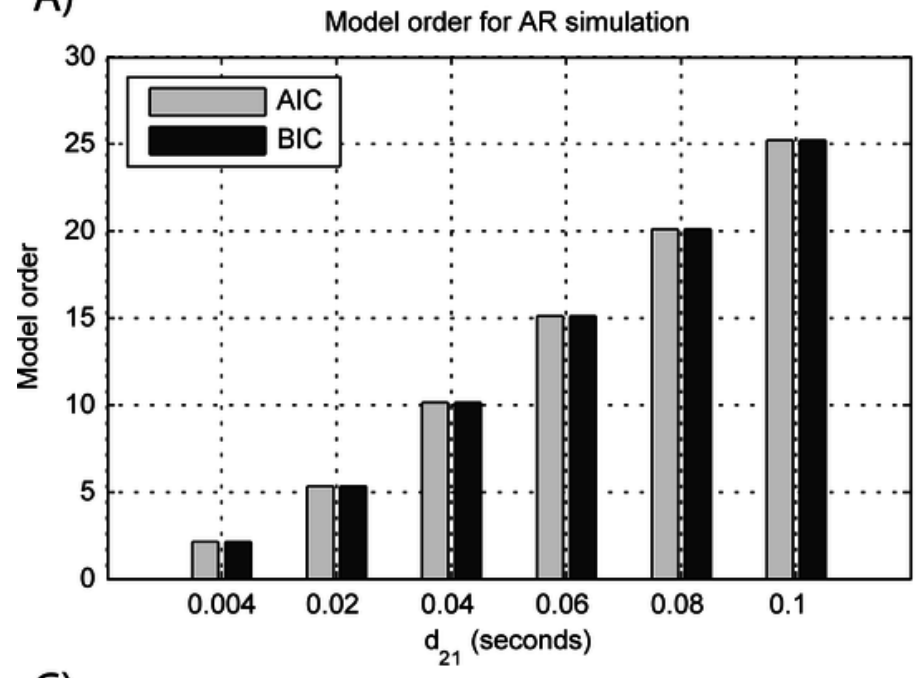

C)

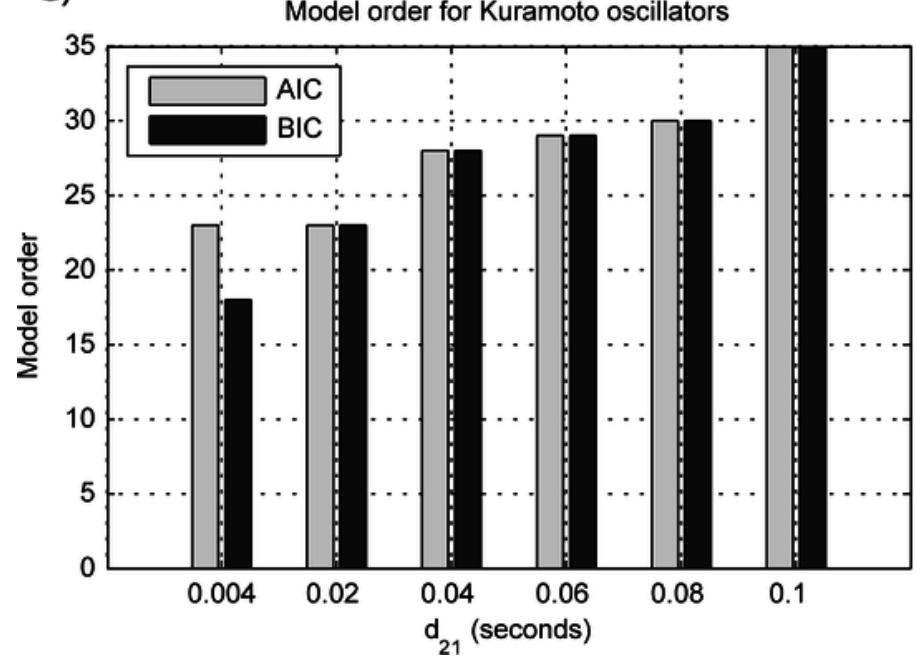

B)

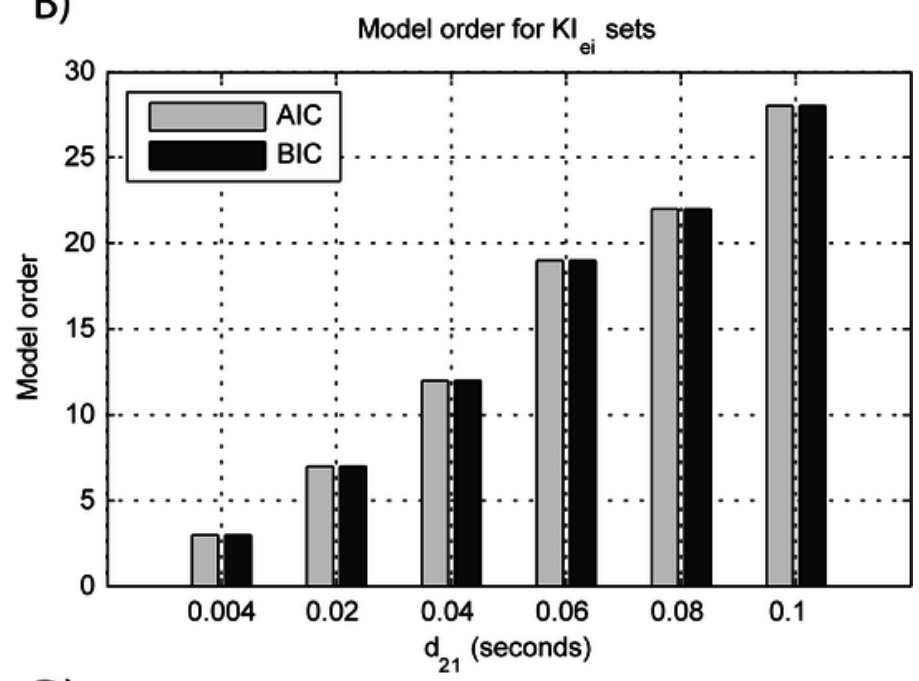

D)

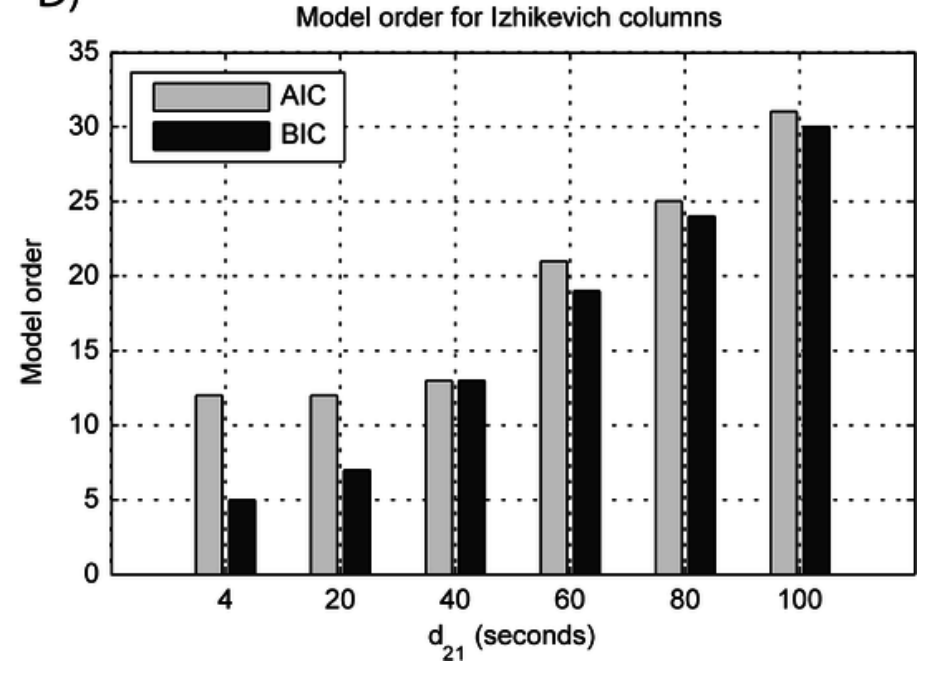


6

GGC DOI after EEG forwarding the time-series from the generative models with varying coupling strengths:

A) MVAR models, B) $K I_{e, i}$ sets, C) Kuramoto oscillators, D, E and F) Izhikevich columns. 

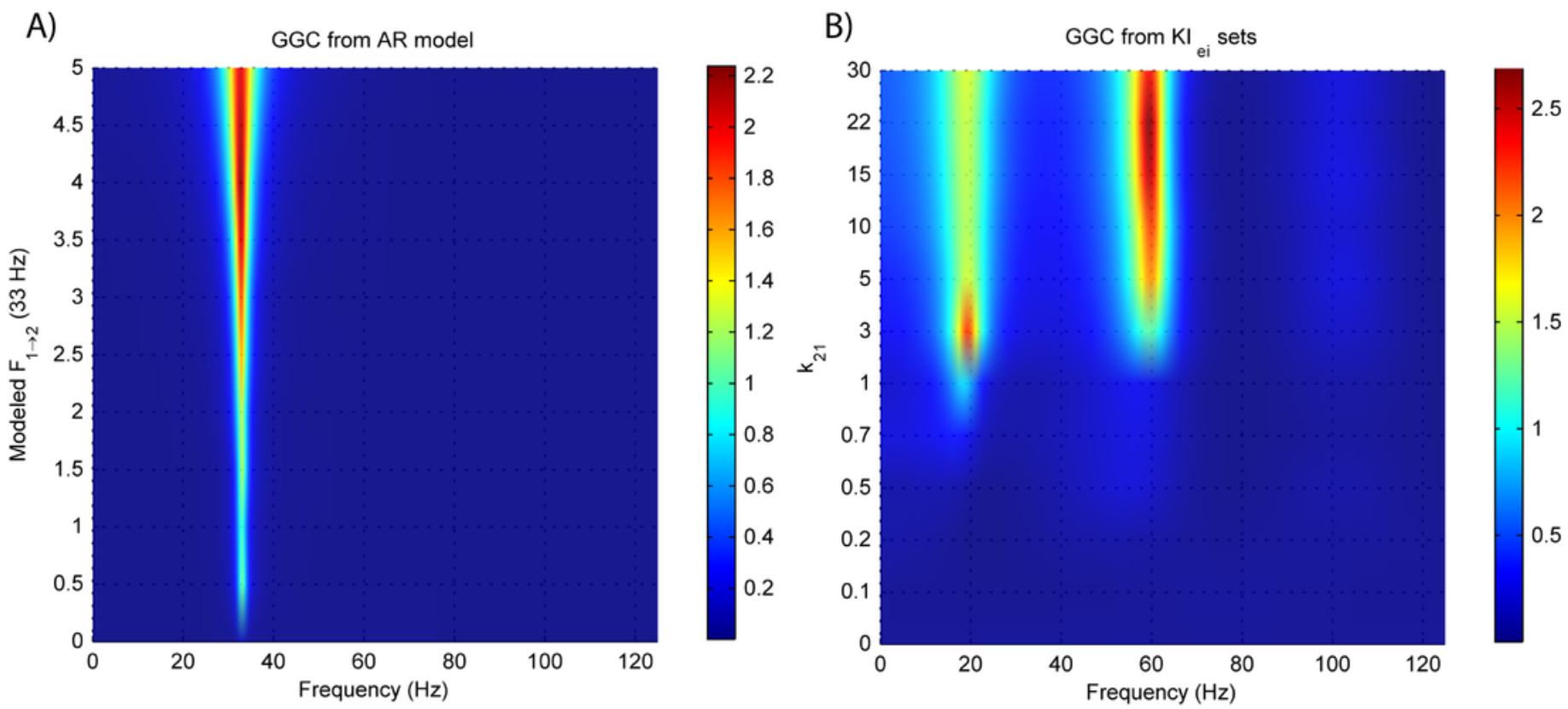

C)

GGC from Kuramoto oscillators
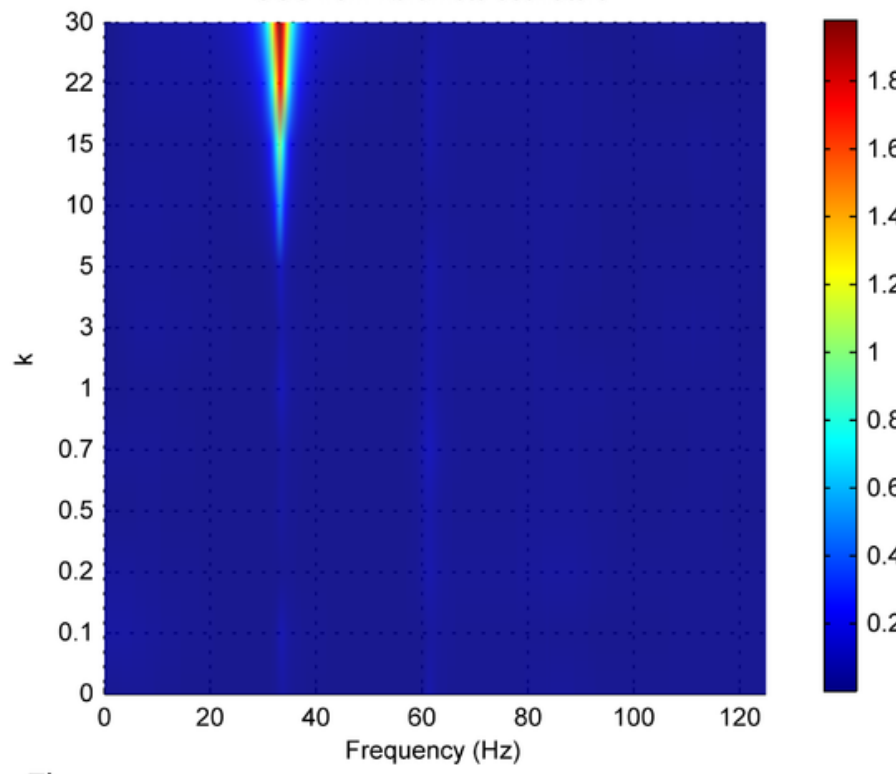

D)

GGC from Izhikevich columns

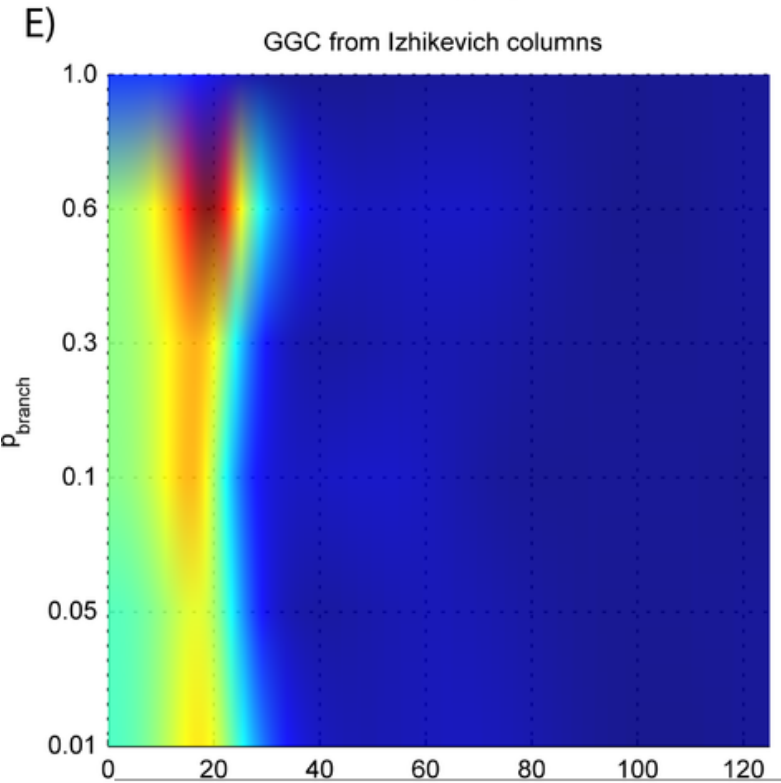

GGC from Izhikevich columns
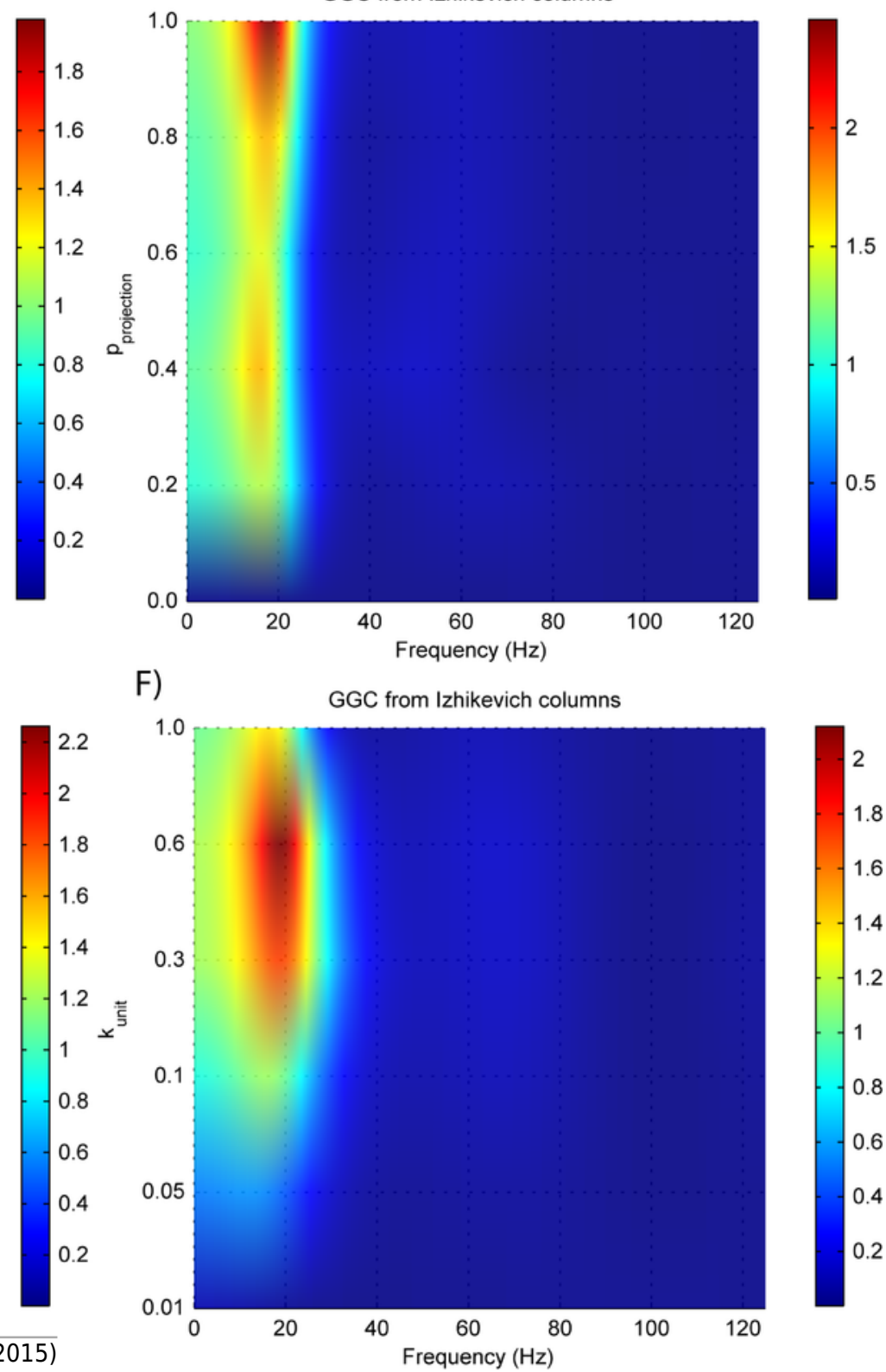

F)

GGC from Izhikevich columns

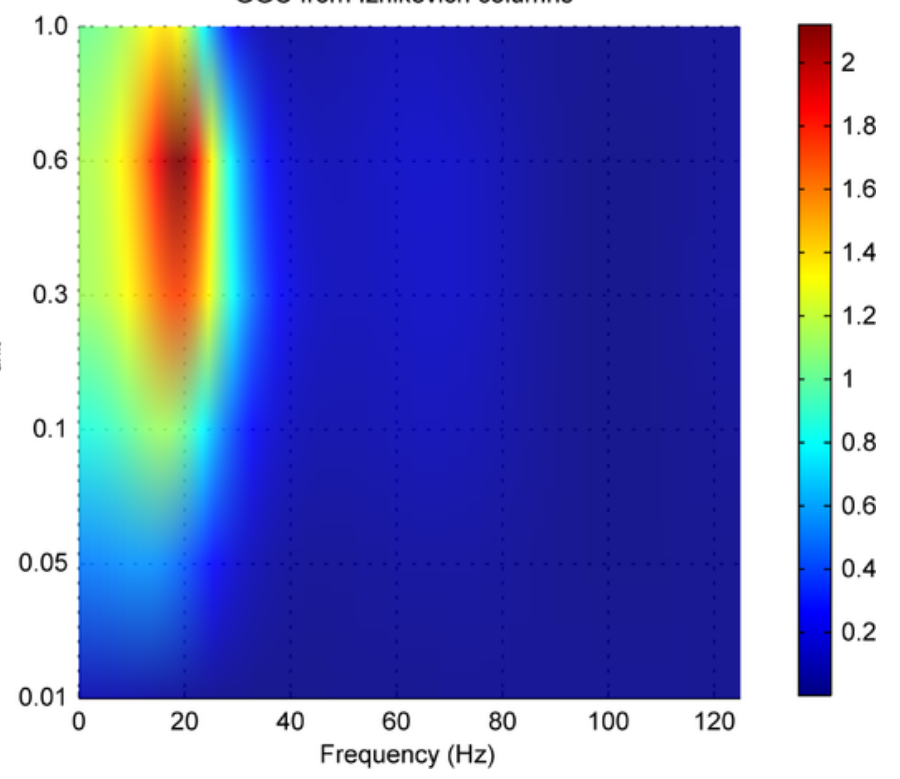




\section{7}

GGC DOI after EEG forwarding the time-series from the generative models with varying interaction delays:

A) MVAR models, B) Kle,i sets, C) Kuramoto oscillators, D) Izhikevich columns.

A)

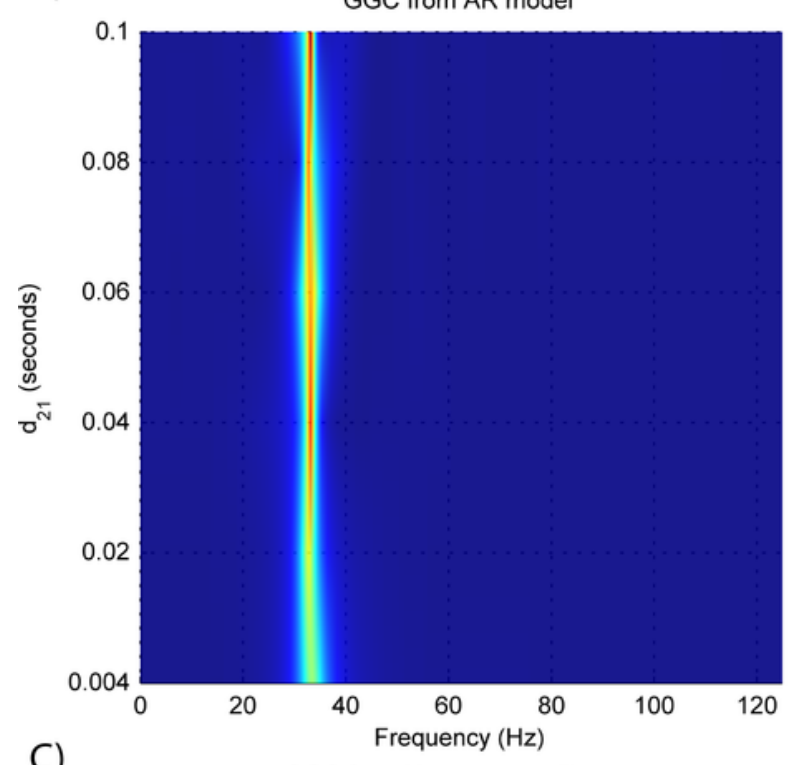

C)

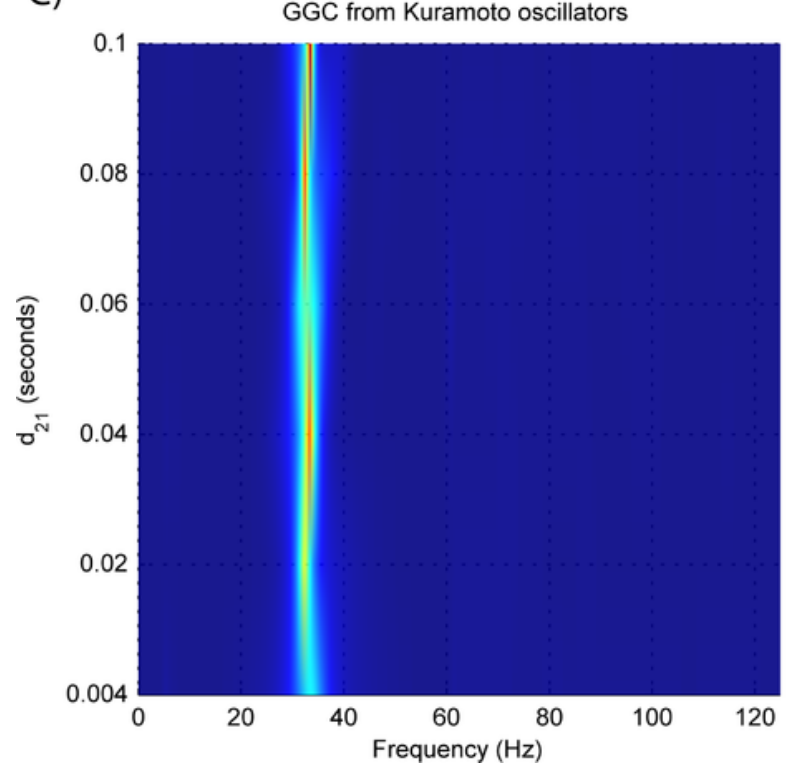

B)

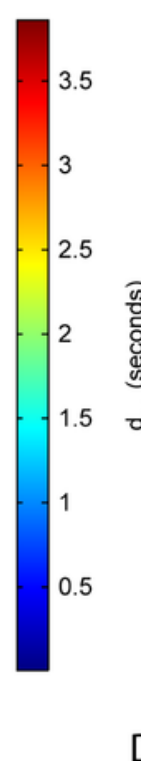
GGC from $\mathrm{KI}_{\mathrm{ei}}$ sets

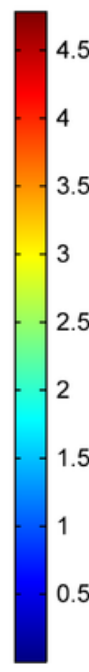

D)
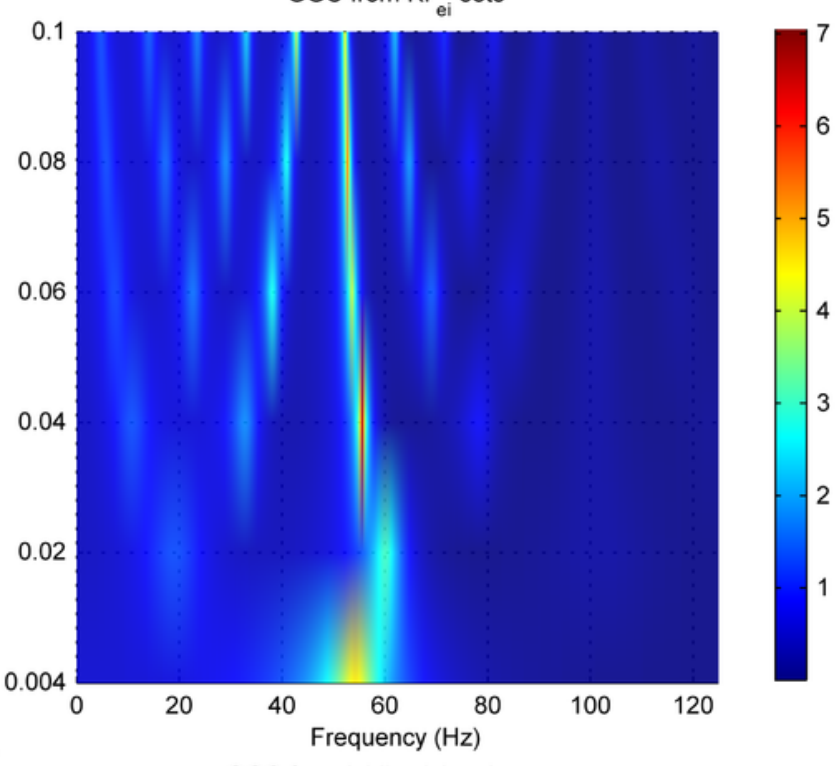

GGC from Izhikevich columns

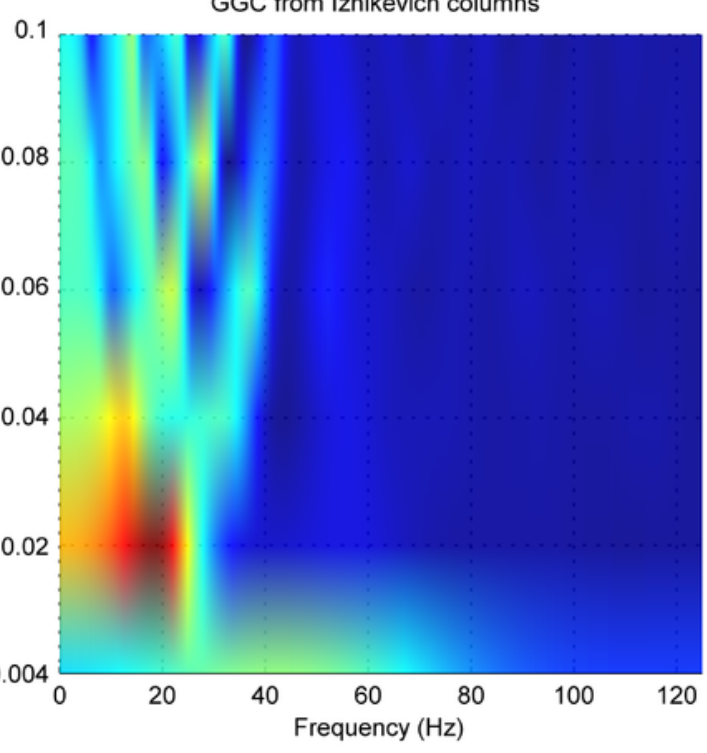


8

Model orders estimated with $\mathrm{BIC}$ and AIC for the time-series simulated with increasing interaction delay after EEG forwarding with the generative models:

A) AR models, B) $K l_{e, i}$ sets, C) Kuramoto oscillators, D) Izhikevich columns. The lagged observations for these interaction delays with $250 \mathrm{HZ}$ sampling rate are $[1,5,10,15,20,25]$.

A)

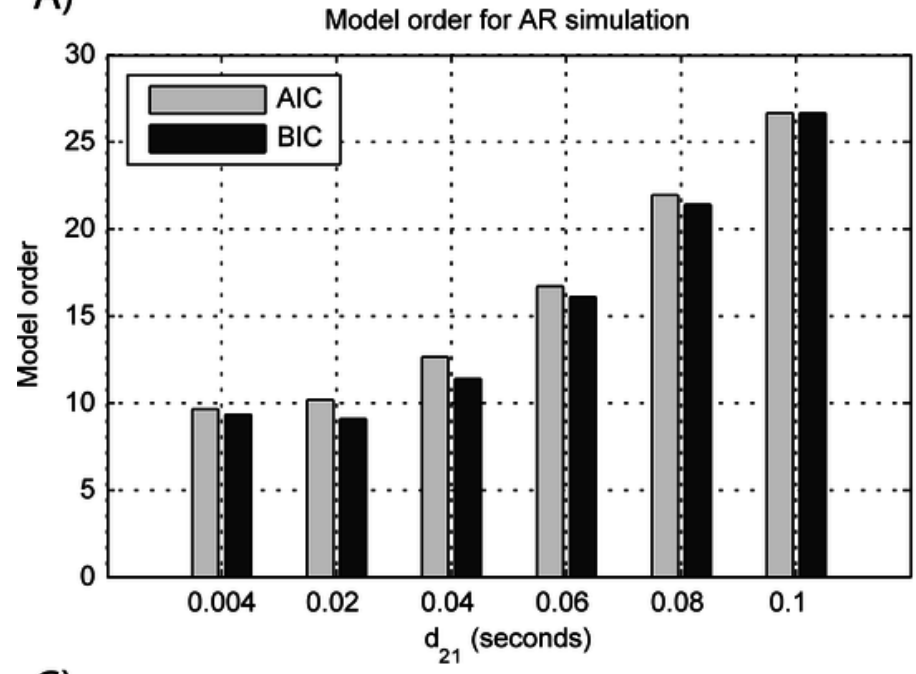

C)

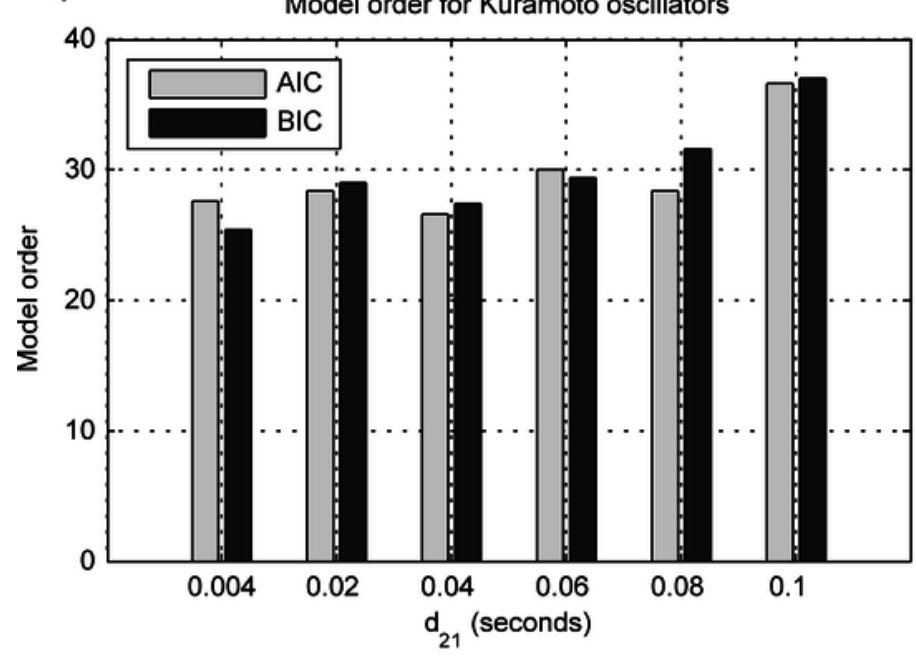

B)

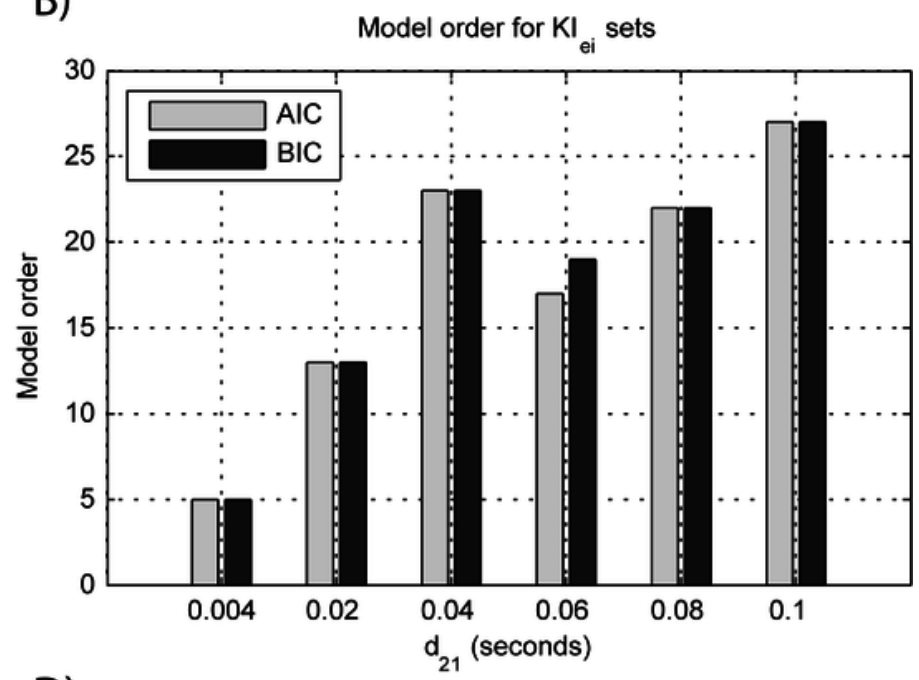

D)

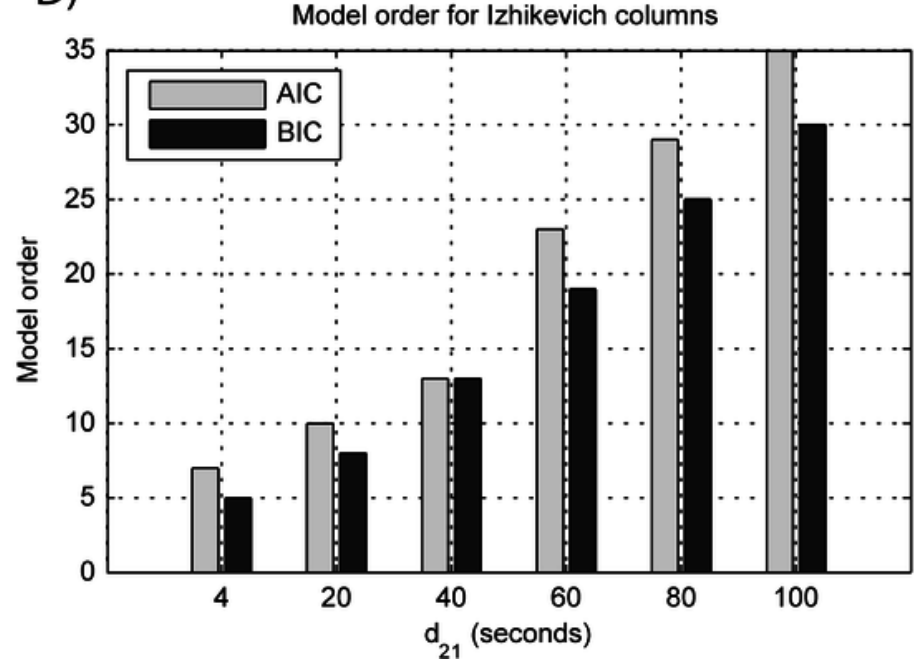


9

GGC DOI in the 0.01-0.1 Hz band after BOLD forward modeling the time-series from the generative models with varying coupling strengths:

A) MVAR models, B) $K I_{e, i}$ sets, C) Kuramoto oscillators, D, E and F) Izhikevich columns.

A)

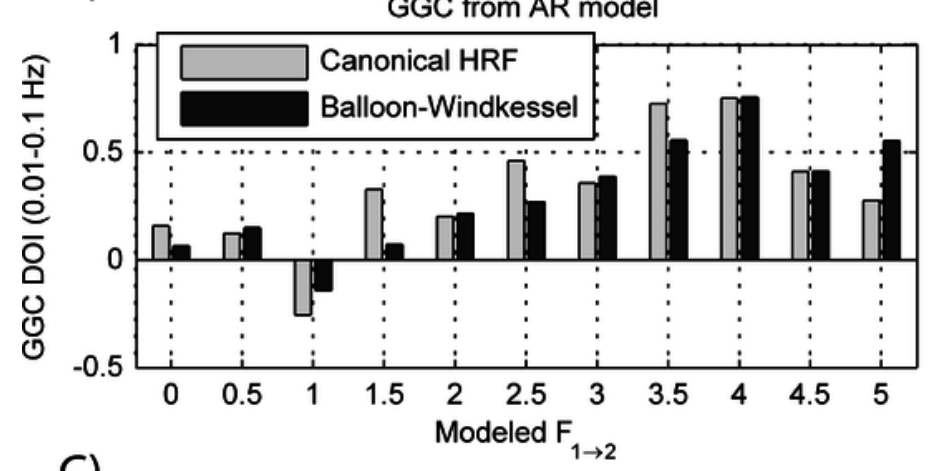

C)

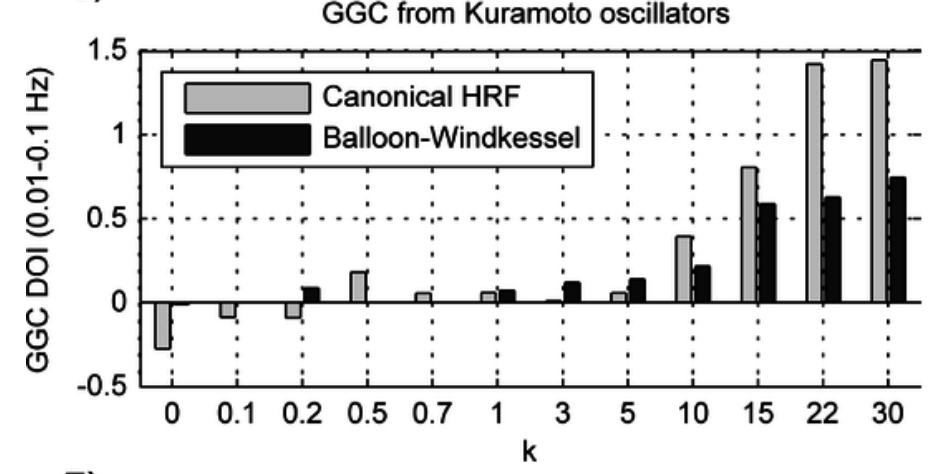

E)

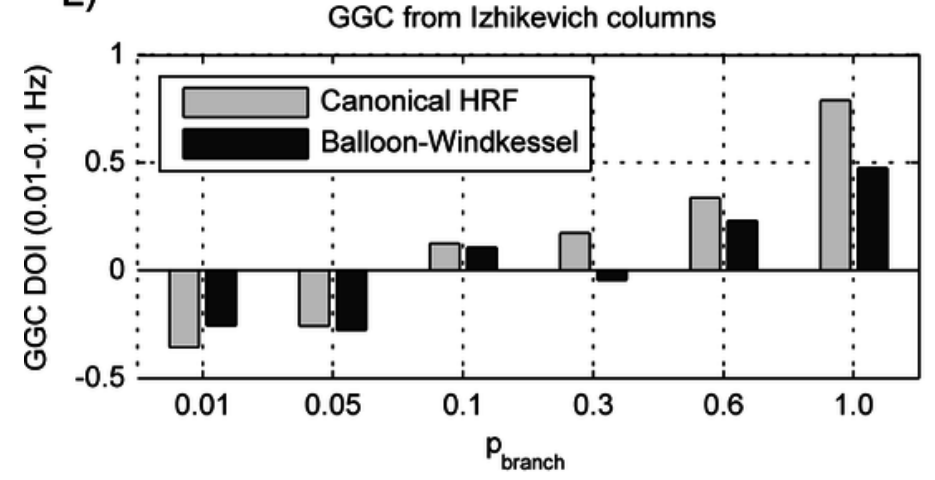

B)

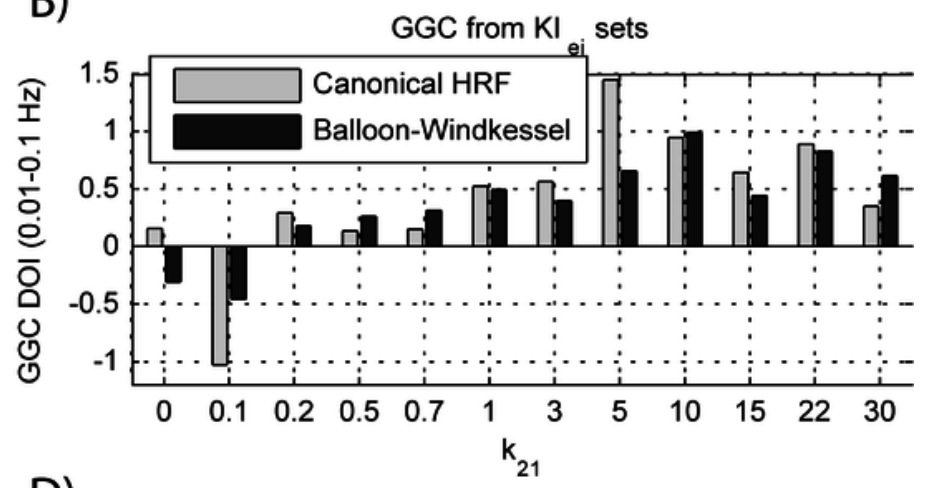

D)

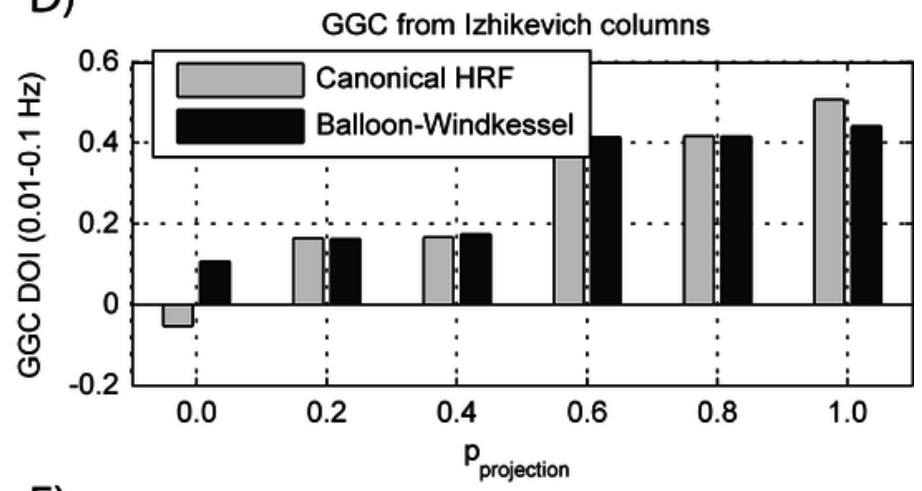

F)

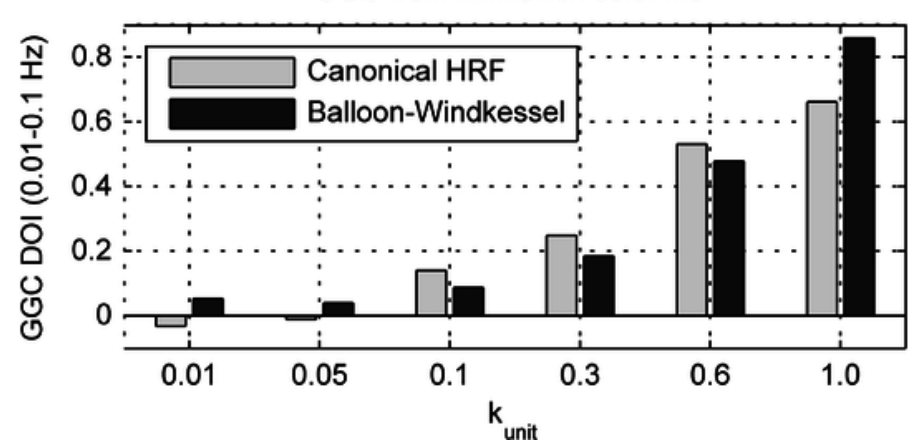




\section{0}

GGC DOI in the $0.01-0.1 \mathrm{~Hz}$ band after BOLD forward modeling the time-series from the generative models with varying interaction delays:

A) MVAR models, B) $\mathrm{Kl}_{e, i}$ sets, C) Kuramoto oscillators, D) Izhikevich columns.

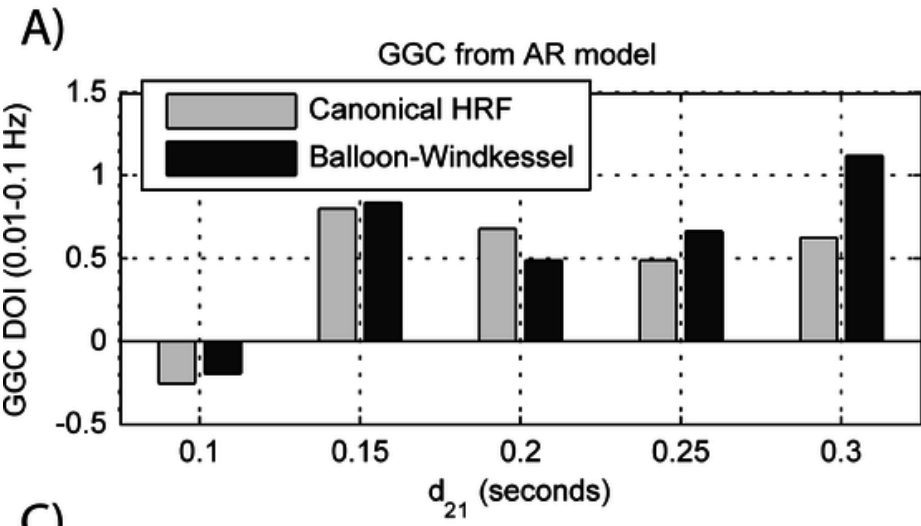

C)

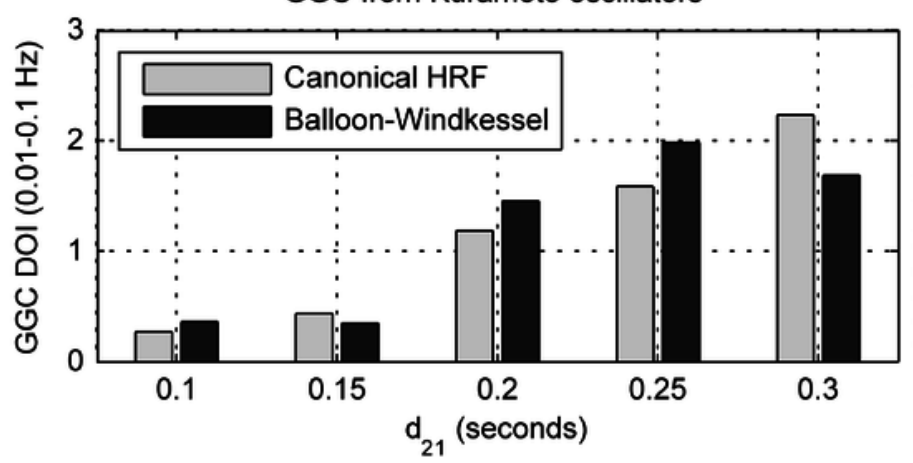

B)

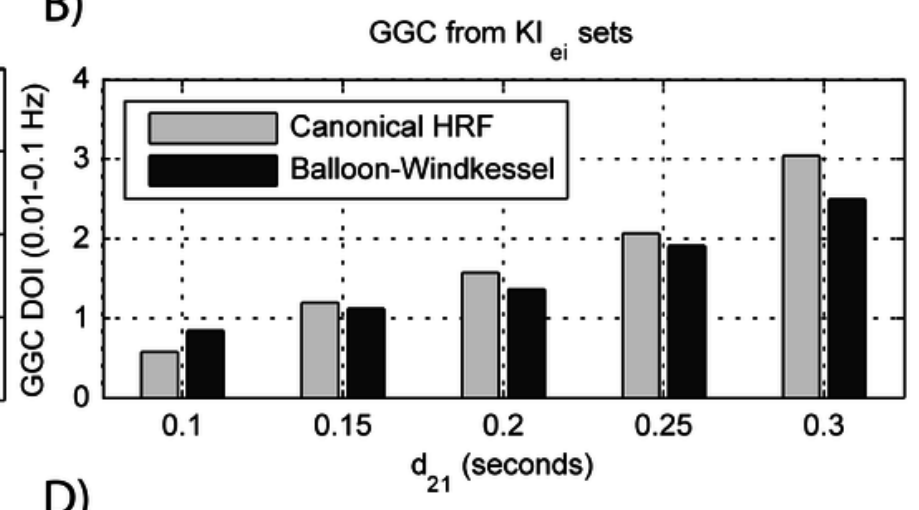

GGC from Izhikevich columns

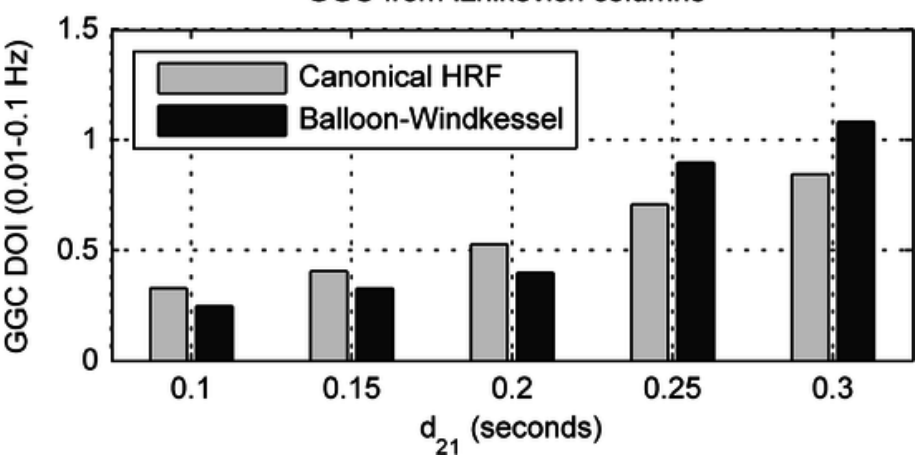




\section{Table $\mathbf{1}$ (on next page)}

Summary of the main results and characteristics for the four generative models. 
2 Table 1: Summary of the main results and characteristics for the four generative models.

\begin{tabular}{|c|c|c|c|c|}
\hline & AR models & $K I_{e, i}$ sets & $\begin{array}{l}\text { Kuramoto } \\
\text { oscillators }\end{array}$ & $\begin{array}{l}\text { Izhikevich } \\
\text { columns }\end{array}$ \\
\hline Causal relations with different strengths & yes & yes & yes & yes \\
\hline Causal relations with different delays & yes & yes & yes & yes \\
\hline Frequency specific causal relations & yes & no & yes & no \\
\hline $\begin{array}{l}\text { Analytic calculation of theoretical } \\
\text { causality }\end{array}$ & yes & no & no & no \\
\hline Neurophysiological model & no & yes & yes & yes \\
\hline $\begin{array}{l}\text { Can simulate different neuronal } \\
\text { dynamics }\end{array}$ & no & no & yes & yes \\
\hline $\begin{array}{l}\text { Causality is preserved after EEG } \\
\text { forwarding }\end{array}$ & yes & yes & yes & yes \\
\hline $\begin{array}{l}\text { Causality is preserved after } \\
\text { hemodynamic forwarding }\end{array}$ & yes & yes & yes & yes \\
\hline Expected computational load & very low & high & average & average \\
\hline
\end{tabular}

